Supporting Information for:

\title{
Catalytic and Highly Regioselective Cross-Coupling of Aromatic C-H Substrates
}

Kami L. Hull and Melanie S. Sanford*

Department of Chemistry, University of Michigan, 930 N. University Avenue, Ann Arbor, MI 48109

I.

General Procedures and Material and Methods

p. S2

II.

Reaction Optimization

p. S3

III.

Experimental Procedures

p. S5

IV.

Mechanistic Studies

p. S22

V.

References

p. S24 


\section{General Procedures and Materials and Methods}

General Procedures: NMR spectra were obtained on a Varian Inova $500\left(499.90 \mathrm{MHz}\right.$ for ${ }^{1} \mathrm{H} ; 125.70$ $\mathrm{MHz}$ for ${ }^{13} \mathrm{C}$ ), a Varian Inova $400\left(399.96 \mathrm{MHz}\right.$ for ${ }^{1} \mathrm{H}$; $100.57 \mathrm{MHz}$ for ${ }^{13} \mathrm{C} ; 376.34 \mathrm{MHz}$ for ${ }^{19} \mathrm{~F}$ ), or a Varian Mercury $300\left(300.07 \mathrm{MHz}\right.$ for ${ }^{1} \mathrm{H}$; $75.45 \mathrm{MHz}$ for ${ }^{13} \mathrm{C}$ NMR; $282.35 \mathrm{MHz}$ for ${ }^{19} \mathrm{~F}$ ) spectrometer. ${ }^{1} \mathrm{H}$ and ${ }^{13} \mathrm{C}$ NMR chemical shifts are reported in parts per million (ppm) relative to TMS, with the residual solvent peak used as an internal reference. ${ }^{19} \mathrm{~F}$ NMR are referenced based on the unified scale, where the frequency of the residual solvent peak in the ${ }^{1} \mathrm{H}$ NMR spectrum acts as the single primary reference. ${ }^{1}{ }^{19} \mathrm{~F}$ NMR are proton coupled. Multiplicities are reported as follows: singlet (s), doublet (d), doublet of doublets (dd), doublet of doublet of doublets (ddd), doublet of triplets (dt), doublet of quartets (dq), triplet $(\mathrm{t})$, triplet of doublets (td), quartet (q), quartet of doublets (qd), and multiplet (m). Unless otherwise indicated, the ${ }^{1} \mathrm{H}$ and ${ }^{13} \mathrm{C}$ NMR spectra were recorded at room temperature.

Materials and Methods: 7,8-Benzoquinoline was obtained from Pfaltz and Bauer, 8-methylquinoline was purchased from Acros, $\mathrm{Ag}_{2} \mathrm{CO}_{3}$ was obtained from Acros, and $\mathrm{Pd}(\mathrm{OAc})_{2}$ was obtained from Pressure Chemical. All were used as received. Benzoquinone was obtained from Acros and was further purified by vacuum sublimation. The arene coupling partners were obtained from commercial sources and used without further purification. $N$-( $m$-Tolyl)-pyrazole was prepared using a Cu-catalyzed $N$-arylation reaction, ${ }^{2}$ and 2-( $m$-tolyl)-pyrimidine and 2-(m-methoxyphenyl)-pyridine were synthesized via Suzuki coupling. ${ }^{3}$ Flash chromatography was performed on EM Science silica gel 60 (0.040-0.063 mm particle size, 230-400 mesh), and thin layer chromatography was performed on Merck TLC plates pre-coated with silica gel $60 \mathrm{~F}_{254}$. Gas chromatography was carried out using a Shimadzu 17A using a Restek Rtx®-5 (Crossbond 5\% diphenyl - 95\% dimethyl polysiloxane; $15 \mathrm{~m}, 0.25 \mathrm{mmID}, 0.25 \mathrm{mmID}, 0.25 \mu \mathrm{m} \mathrm{df}$ ) column. HPLC was performed on a Varian ProStar 210 HPLC using Waters $\mu$ Porasil ${ }^{\circledR} 10 \mu \mathrm{m}$ silica $(19 \mathrm{x}$ $300 \mathrm{~mm}$ ) columns. Control reactions (in the absence of Pd catalyst) were run for each substrate, and showed none of the desired coupled product under our standard reaction conditions. All GC and isolated yields are the average of two reactions. 


\section{Reaction Optimization}

Table S1. Variation of Electron Deficient Olefin Derivative

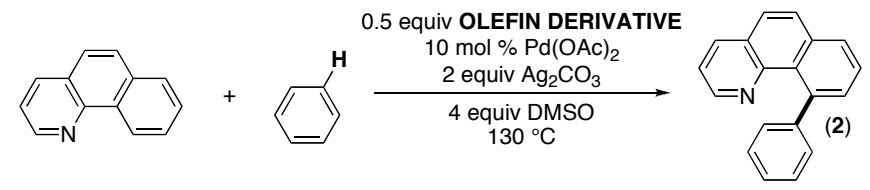

\begin{tabular}{|c|c|c|}
\hline Entry & Olefin Derivative & GC Yield 2 \\
\hline 1 & benzoquinone & $89 \%$ \\
2 & 2-methylbenzoquinone & $80 \%$ \\
3 & 2,6-dimethylbenzoquinone & $54 \%$ \\
4 & duroquinone & $53 \%$ \\
5 & 2,6-dichlorobenzoquinone & $36 \%$ \\
6 & 2,5-dichlorobenzoquinone & $43 \%$ \\
7 & tetrachlorobenzoquinone & $2 \%$ \\
8 & tetrafluorobenzoquinone & $0 \%$ \\
9 & tetracyanoethylene & $0 \%$ \\
10 & trans- $\beta$-nitrostyrene & $15 \%$ \\
\hline
\end{tabular}

Table S2. Variation of Equivalents of $\mathrm{C}_{6} \mathrm{H}_{6}$ (constant [DMSO])

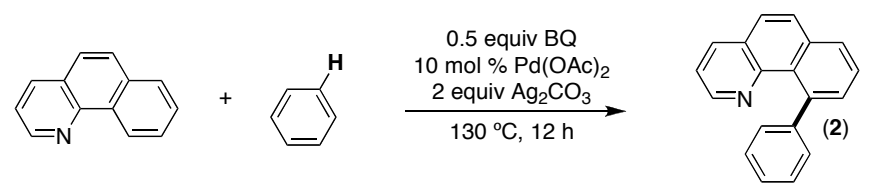

\begin{tabular}{|c|c|c|c|c|c|}
\hline Entry & volume $\mathrm{C}_{6} \mathrm{H}_{6}$ & equiv $\mathrm{C}_{6} \mathrm{H}_{6}$ & volume DMSO & equiv DMSO & GC Yield 2 \\
\hline 1 & $0.19 \mathrm{~mL}$ & 5 & $9 \mu \mathrm{L}$ & 0.3 & $18 \%^{\mathrm{a}}$ \\
2 & $0.38 \mathrm{~mL}$ & 10 & $17 \mu \mathrm{L}$ & 0.6 & $45 \%$ \\
3 & $0.77 \mathrm{~mL}$ & 20 & $34 \mu \mathrm{L}$ & 1.1 & $63 \%$ \\
4 & $1.54 \mathrm{~mL}$ & 40 & $69 \mu \mathrm{L}$ & 2.3 & $71 \%$ \\
5 & $3.07 \mathrm{~mL}$ & 80 & $138 \mu \mathrm{L}$ & 4.5 & $87 \%$ \\
\hline
\end{tabular}

${ }^{\mathrm{a}} \sim 4 \%$ of Bzq-Bzq and $\sim 54 \%$ of unreacted Bzq was observed as determined by GC and ${ }^{1} \mathrm{H}$ NMR spectroscopy. 
Table S3. Variation of Oxidant

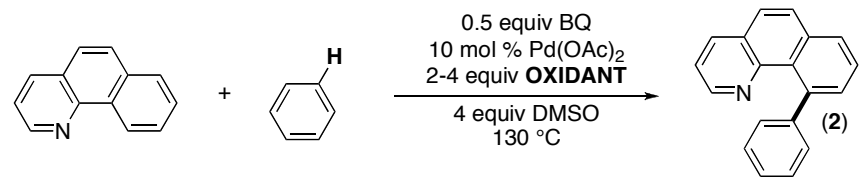

\begin{tabular}{|c|c|c|c|}
\hline Entry & Oxidant & equiv & GC Yield 2 \\
\hline 1 & none & 0 & $10 \%$ \\
\hline 2 & benzoquinone & 4 & $9 \%$ \\
\hline 3 & $\mathrm{~K}_{2} \mathrm{~S}_{2} \mathrm{O}_{8}$ & 4 & $1 \%$ \\
\hline 4 & Oxone & 4 & $0 \%$ \\
\hline 5 & $\mathrm{CuCl}_{2}$ & 4 & $0 \%$ \\
\hline 6 & $\mathrm{Cu}(\mathrm{OAc})_{2} \cdot \mathrm{H}_{2} \mathrm{O}$ & 4 & $53 \%$ \\
\hline 7 & $\mathrm{CuO}$ & 4 & $8 \%$ \\
\hline 8 & $\mathrm{Ag}\left(\mathrm{O}_{2} \mathrm{CCF}_{3}\right)$ & 4 & $0 \%$ \\
\hline 9 & AgOTf & 4 & $0 \%$ \\
\hline 10 & $\mathrm{Ag}_{2} \mathrm{O}$ & 2 & $15 \%$ \\
\hline 11 & $\mathrm{AgOAc} \cdot \mathrm{H}_{2} \mathrm{O}$ & 4 & $38 \%$ \\
\hline 12 & $\mathrm{Ag}_{2} \mathrm{CO}_{3}$ & 2 & $89 \%$ \\
\hline
\end{tabular}

Table S4. Variation of Sulfoxide

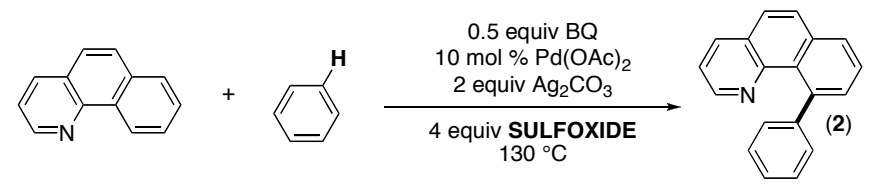

\begin{tabular}{|c|c|c|}
\hline Entry & Sulfoxide Derivative & GC Yield \\
\hline 1 & none & $74 \%$ \\
2 & DMSO & $89 \%$ \\
3 & tetramethylene sulfoxide & $86 \%$ \\
4 & diphenyl sulfoxide & $75 \%$ \\
5 & dibenzyl sulfoxide & $52 \%$ \\
6 & 1,2-bis(benzylsulfinyl)ethane & $33 \%$ \\
\hline
\end{tabular}




\section{Experimental Procedures}

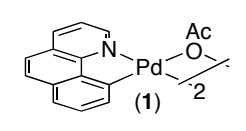

1: Palladacycle 1 was prepared as reported in the literature. ${ }^{4}$

$\mathrm{L} \sim \mathrm{C}_{\mathrm{Ar}}-\mathrm{H} \quad \mathrm{Ar}-\mathrm{H} \longrightarrow \quad \mathrm{L} \sim \mathrm{C}_{\mathrm{Ar}}-\mathrm{Ar}$

General Procedure: $\mathrm{Pd}(\mathrm{OAc})_{2}(9.6 \mathrm{mg}, 0.048 \mathrm{mmol}, 10 \mathrm{~mol} \%)$, benzoquinone $(23.7 \mathrm{mg}, 0.22$ mmol, 0.5 equiv), $\mathrm{Ag}_{2} \mathrm{CO}_{3}$ (237.2 $\mathrm{mg}, 0.86 \mathrm{mmol}, 2$ equiv), and the directed $\mathrm{C}-\mathrm{H}$ activation substrate $\left(\mathrm{L} \sim \mathrm{C}_{\mathrm{Ar}}-\mathrm{H}\right)$ (if solid) $(0.43 \mathrm{mmol}, 1$ equiv) were weighed into a $20 \mathrm{~mL}$ scintillation vial equipped with a 15 x $5 \mathrm{~mm}$ egg-shaped Teflon stirbar. The arene $(\mathrm{Ar}-\mathrm{H})(3.75 \mathrm{~mL})$ was added, followed by $\mathrm{L} \sim \mathrm{C}_{\mathrm{Ar}}-\mathrm{H}$ (if oil) $(0.43 \mathrm{mmol}, 1$ equiv), and finally, $125 \mu \mathrm{L}$ of DMSO (1.45 mmol, 3.4 equiv). The resulting mixture was sealed with a Teflon-lined cap and heated in an aluminum reaction block with vigorous stirring (IKA RCT Basic Hot Plate Stirrer with stirring set at 11). Note: consistent stirring was critical for the reproducibility of these reactions. Reactions run in the absence of stirring showed a significant decrease in yield. ${ }^{*}$ The reaction mixtures generally turned grey/brown upon heating. The reaction was cooled to room temperature, filtered through a plug of silica, and the silica was washed with copious EtOAc $(150 \mathrm{~mL})$. The filtrate was concentrated and then evaporated to dryness under high vacuum, and the resulting oils/solids were purified by column chromatography.

* If the reactions were heated in an oil bath, the best results were obtained when they were prestirred prior to heating and the stirring rate was monitored throughout.

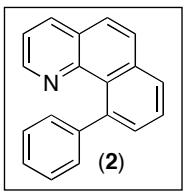

Product 2. $\mathrm{L} \sim \mathrm{C}_{\mathrm{Ar}}-\mathrm{H}=7,8$-benzo[ $\left.h\right]$ quinoline; $\mathrm{Ar}-\mathrm{H}=$ benzene; temperature $=130{ }^{\circ} \mathrm{C}$. The product was isolated as a white crystalline solid $\left(98 \mathrm{mg}, 89 \%\right.$ yield, $\mathrm{mp}=90.6-92.5{ }^{\circ} \mathrm{C}, \mathrm{rf}=0.11$ in $98 \%$ hexanes $/ 2 \%$ EtOAc). ${ }^{1} \mathrm{H}$ NMR $\left(400 \mathrm{MHz}\right.$, acetone- $\left.d_{6}\right): \delta 8.36\left(\mathrm{H}^{1}, \mathrm{dd}, J=4.0,2.0 \mathrm{~Hz}\right.$, $1 \mathrm{H}), 8.21\left(\mathrm{H}^{3}, \mathrm{dd}, J=8.0,1.6 \mathrm{~Hz}, 1 \mathrm{H}\right), 7.99\left(\mathrm{H}^{8}, \mathrm{dd}, J=7.8,1.4 \mathrm{~Hz}, 1 \mathrm{H}\right), 7.93\left(\mathrm{H}^{4}, \mathrm{~d}, J=8.8\right.$ 
$\mathrm{Hz}, 1 \mathrm{H}), 7.79\left(\mathrm{H}^{5}, \mathrm{~d}, J=8.8 \mathrm{~Hz}, 1 \mathrm{H}\right), 7.69\left(\mathrm{H}^{7}, \mathrm{dd}, J=8.0,7.2 \mathrm{~Hz}, 1 \mathrm{H}\right), 7.48\left(\mathrm{H}^{6}, \mathrm{dd}, J=7.4\right.$, $1.4 \mathrm{~Hz}, 1 \mathrm{H}), 7.40-7.25\left(\mathrm{H}^{2}, \mathrm{H}^{9}-\mathrm{H}^{11}\right.$, multiple peaks, $\left.6 \mathrm{H}\right) ;{ }^{13} \mathrm{C}$ NMR $\left(100 \mathrm{MHz}\right.$, acetone- $\left.d_{6}\right): \delta$ 147.61, 147.47, 147.43, 142.65, 136.22, 135.98, 132.22, 129.71, 129.50, 129.11, 128.95, 128.18, $128.05,127.95,126.98,126.36,122.19$. HRMS ESI with Formic Acid $(\mathrm{m} / \mathrm{z}):[\mathrm{M}+\mathrm{H}]^{+}$calcd for $\mathrm{C}_{19} \mathrm{H}_{14} \mathrm{~N}, 256.1126$; found, 256.1115 .

Note: The absence of $\mathrm{Ph}-\mathrm{Ph}$ in the crude reaction mixture was determined by GC analysis versus an authentic sample of biphenyl. The absence of the Bzq oxidative coupling product (Bzq-Bzq) was determined by ${ }^{1} \mathrm{H}$ NMR spectroscopy of the crude reaction mixture, where the diagnostic peak at $6.41 \mathrm{ppm}$ (see below) was not observed.
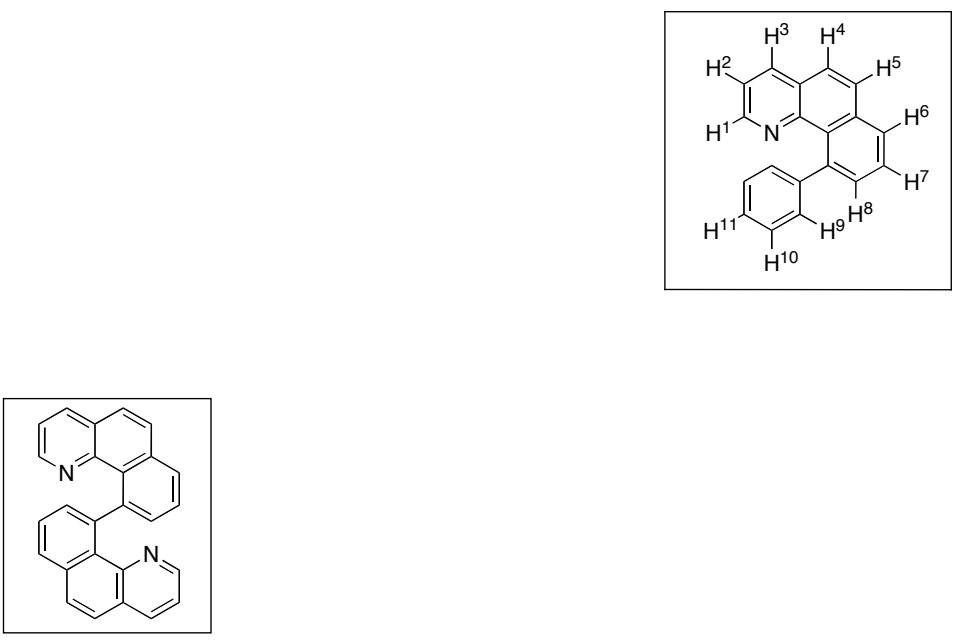

An authentic sample of the Bzq oxidative dimerization product was obtained according to the procedure in ref 5 . The characterization data for this compound are as follows: ${ }^{1} \mathrm{H}$ NMR (400 MHz, benzene- $\left.d_{6}\right): \delta 7.86\left(\mathrm{H}^{8}, \mathrm{dd}, J=7.6,1.6 \mathrm{~Hz}, 2 \mathrm{H}\right), 7.83\left(\mathrm{H}^{1}, \mathrm{dd}, J=4.2,1.8 \mathrm{~Hz}, 2 \mathrm{H}\right), 7.76$ $\left(\mathrm{H}^{4}, \mathrm{~d}, J=8.8 \mathrm{~Hz}, 2 \mathrm{H}\right), 7.60-7.51\left(\mathrm{H}^{3}, \mathrm{H}^{7}\right.$, multiple peaks, $\left.4 \mathrm{H}\right), 7.37\left(\mathrm{H}^{6}, \mathrm{dd}, J=8.0,1.2 \mathrm{~Hz}\right.$, $2 \mathrm{H}), 7.33\left(\mathrm{H}^{5}, \mathrm{~d}, J=8.8 \mathrm{~Hz}, 2 \mathrm{H}\right), 6.41\left(\mathrm{H}^{2}, \mathrm{dd}, J=8.0,4.4 \mathrm{~Hz}, 2 \mathrm{H}\right) ;{ }^{13} \mathrm{C}\left\{{ }^{1} \mathrm{H}\right\} \mathrm{NMR}\left(\mathrm{CDCl}_{3}\right): \delta$ 147.41, 146.67, 145.99, 134.67, 134.33, 130.39, 128.75, 128.63, 127.20, 126.63, 125.21, 120.27 ( 2 carbon peaks coincidentally overlap); MS-electrospray $(\mathrm{m} / \mathrm{z})$ : $[\mathrm{M}+\mathrm{H}]^{+}$calcd for $\mathrm{C}_{26} \mathrm{H}_{17} \mathrm{~N}_{2}$, 357.1392; Found, 357.1396.

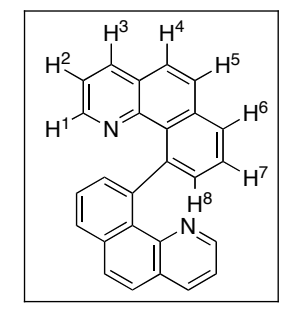




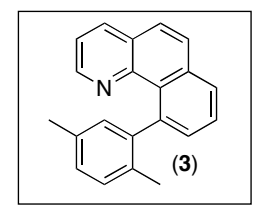

Product 3. Reaction was run at five times the scale of the general procedure above. $\mathrm{L} \sim \mathrm{C}_{\mathrm{Ar}}-\mathrm{H}=$ 7,8-benzo $[h]$ quinoline; $\mathrm{Ar}-\mathrm{H}=p$-xylene; temperature $=150{ }^{\circ} \mathrm{C}$. The product was isolated as a clear oil (22 mg, $5 \%$ yield, $\mathrm{rf}=0.24$ in $98 \%$ hexanes $/ 2 \%$ EtOAc). Trace amounts $(<5 \%)$ of the Bzq-Bzq dimer were observed in the ${ }^{1} \mathrm{H}$ NMR spectrum of the crude reaction mixture. ${ }^{1} \mathrm{H}$ NMR $\left(400 \mathrm{HMz}, \mathrm{CDCl}_{3}\right): \delta 8.46\left(\mathrm{H}^{1}, \mathrm{dd}, J=4.2,1.8 \mathrm{~Hz}, 1 \mathrm{H}\right), 8.08\left(\mathrm{H}^{3}, \mathrm{dd}, J=8.2,1.8 \mathrm{~Hz}, 1 \mathrm{H}\right), 7.94$ $\left(\mathrm{H}^{8}, \mathrm{dd}, J=8.0,1.2 \mathrm{~Hz}, 1 \mathrm{H}\right), 7.88\left(\mathrm{H}^{4}, \mathrm{~d}, J=8.8 \mathrm{~Hz}, 1 \mathrm{H}\right), 7.74-7.67\left(\mathrm{H}^{5}, \mathrm{H}^{7}\right.$, multiple peaks, $2 \mathrm{H}), 7.48\left(\mathrm{H}^{6}, \mathrm{dd}, J=7.2,1.2 \mathrm{~Hz}, 1 \mathrm{H}\right), 7.32\left(\mathrm{H}^{2}, \mathrm{dd}, J=8.0,4.0 \mathrm{~Hz}, 1 \mathrm{H}\right), 7.16-7.09\left(\mathrm{H}^{11}, \mathrm{H}^{12}\right.$ multiple peaks, $2 \mathrm{H}), 7.04\left(\mathrm{H}^{9}, \mathrm{bs}, 1 \mathrm{H}\right), 2.39\left(\mathrm{H}^{13}, \mathrm{~s}, 3 \mathrm{H}\right), 1.80\left(\mathrm{H}^{10}, \mathrm{~s}, 3 \mathrm{H}\right) \cdot{ }^{13} \mathrm{C}\left\{{ }^{1} \mathrm{H}\right\}$ NMR $(100$ $\left.\mathrm{MHz}, \mathrm{CDCl}_{3}\right): \delta 147.45,147.01,146.19,141.15,134.99,134.57,134.30,132.76,130.81$, $129.43,128.61,128.51,128.42,127.76,127.18,126.88,126.49,125.74,120.91,21.15,19.67$; MS-electrospray $(\mathrm{m} / \mathrm{z})$ : $[\mathrm{M}+\mathrm{H}]^{+}$calcd for $\mathrm{C}_{21} \mathrm{H}_{18} \mathrm{~N}, 284.1439$; Found, 284.1437.
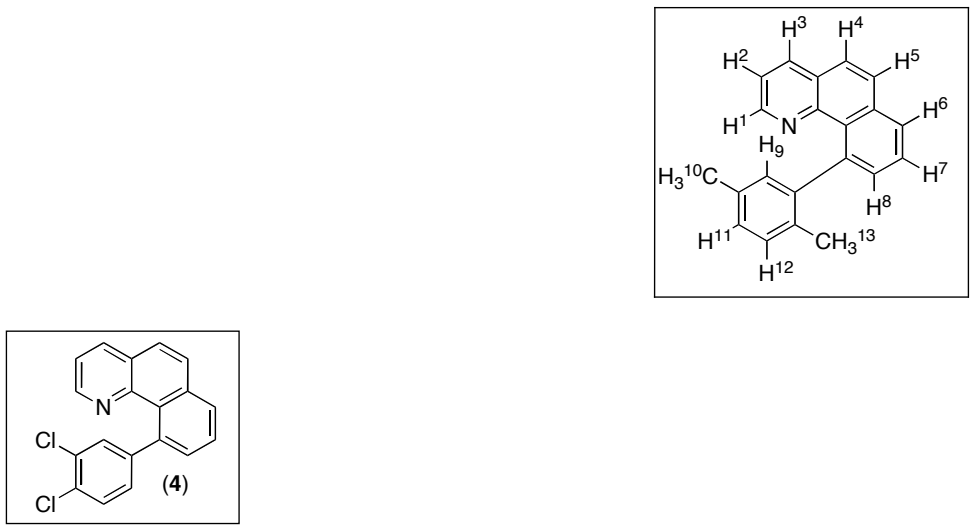

Product 4. $\mathrm{L} \sim \mathrm{C}_{\mathrm{Ar}}-\mathrm{H}=7,8$-benzo[ $\left.h\right]$ quinoline; $\mathrm{Ar}-\mathrm{H}=o$-dichlorobenzene; temperature $=130$ ${ }^{\circ} \mathrm{C}$. The product was isolated as a pale yellow solid $\left(130 \mathrm{mg}, 93 \%\right.$ yield, $\mathrm{mp}=117.8-121.7^{\circ} \mathrm{C}$, rf $=0.22$ in $98 \%$ hexanes $/ 2 \%$ EtOAc) ${ }^{1} \mathrm{H}$ NMR $\left(500 \mathrm{MHz}\right.$, acetone- $\left.d_{6}\right): \delta 8.44\left(\mathrm{H}^{1}, \mathrm{dd}, J=4.0,2.0\right.$ $\mathrm{Hz}, 1 \mathrm{H}), 8.28\left(\mathrm{H}^{3}, \mathrm{dd}, J=8.0,2.0 \mathrm{~Hz}, 1 \mathrm{H}\right), 8.06\left(\mathrm{H}^{8}, \mathrm{dd}, J=7.8,1.3 \mathrm{~Hz}, 1 \mathrm{H}\right), 7.97\left(\mathrm{H}^{4}, \mathrm{~d}, J=\right.$ $8.5 \mathrm{~Hz}, 1 \mathrm{H}), 7.85\left(\mathrm{H}^{5}, \mathrm{~d}, J=8.5 \mathrm{~Hz}, 1 \mathrm{H}\right), 7.73\left(\mathrm{H}^{7}, \mathrm{dd}, J=8.0,7.5 \mathrm{~Hz}, 1 \mathrm{H}\right), 7.52\left(\mathrm{H}^{10}, \mathrm{~d}, J=8.0\right.$ $\mathrm{Hz}, 1 \mathrm{H}), 7.49\left(\mathrm{H}^{6}, \mathrm{dd}, J=7.3,1.3 \mathrm{~Hz}, 1 \mathrm{H}\right), 7.45\left(\mathrm{H}^{11}, \mathrm{~d}, J=2.0 \mathrm{~Hz}, 1 \mathrm{H}\right), 7.45\left(\mathrm{H}^{2}, \mathrm{dd}, J=8.0\right.$, $4.5 \mathrm{~Hz}, 1 \mathrm{H}), 7.21\left(\mathrm{H}^{9}, \mathrm{dd}, J=8.0,2.0 \mathrm{~Hz}, 1 \mathrm{H}\right) ;{ }^{13} \mathrm{C}$ NMR $\left(125 \mathrm{MHz}\right.$, acetone- $\left.d_{6}\right): \delta 148.05$, 147.89 , 147.04, 139.70, 136.61, 136.04, 132.01, 131.42, 131.41, 130.13, 129.96, 129.85, 129.73, 
129.43, 129.13, 128.40, 128.14, 127.31, 122.58. HRMS ESI with Formic Acid (m/z): $[\mathrm{M}+\mathrm{H}]^{+}$ calcd for $\mathrm{C}_{19} \mathrm{H}_{12} \mathrm{NCl}_{2}, 324.0347$; found, 324.0337 .

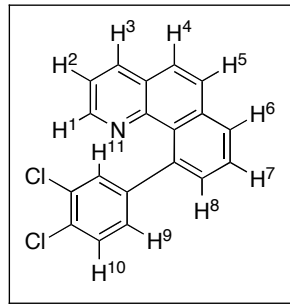

\section{Confirmation of regioselectivity (assignments based on COSY analysis)}
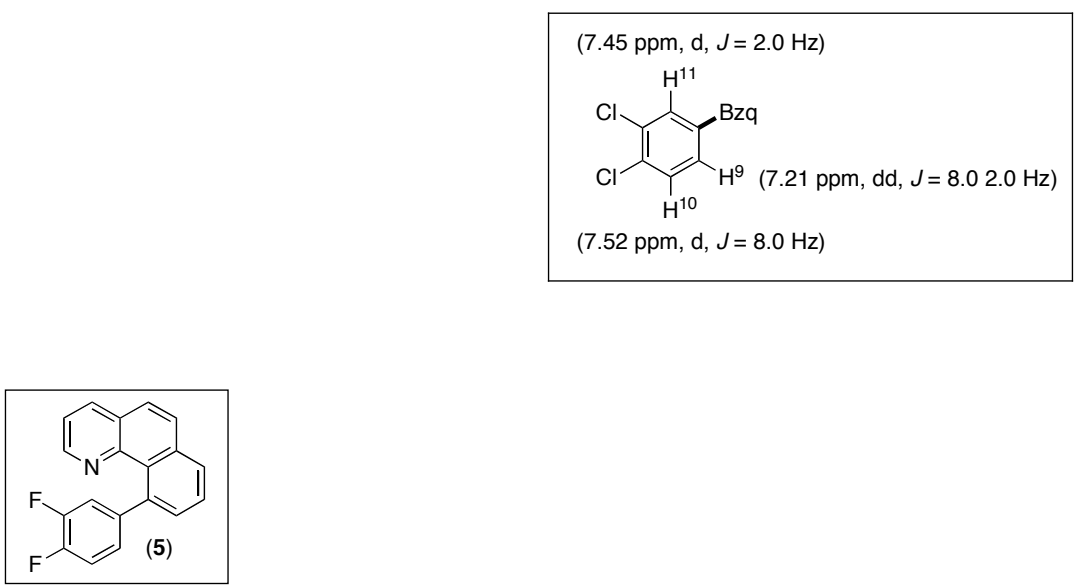

Product 5. $\mathrm{L} \sim \mathrm{C}_{\mathrm{Ar}}-\mathrm{H}=7,8$-benzo[ $[h]$ quinoline; $\mathrm{Ar}-\mathrm{H}=o$-difluorobenzene; temperature $=150$ ${ }^{\circ} \mathrm{C}$. The crude product was a 10:1 mixture of regioisomers (as determined by crude ${ }^{19} \mathrm{~F}$ NMR spectroscopy of the reaction mixture). A pure sample of the major regioisomer was isolated as a pale orange viscous oil $\left(61 \mathrm{mg}, 49 \%\right.$ yield, $\mathrm{mp}=123.2-125.2{ }^{\circ} \mathrm{C}, \mathrm{rf}=0.17$ in $98 \%$ hexanes $/ 2 \%$ EtOAc). Trace amounts $(<5 \%)$ of the Bzq-Bzq dimer were observed in the ${ }^{1} \mathrm{H}$ NMR spectrum of the crude reaction mixture. The structure of the major regioisomer was confirmed by GCOSY and HSQC. ${ }^{1} \mathrm{H}$ NMR (400 MHz, acetone- $\left.d_{6}\right): \delta 8.46\left(\mathrm{H}^{1}, \mathrm{dd}, J=4.4,2.0 \mathrm{~Hz}, 1 \mathrm{H}\right), 8.28\left(\mathrm{H}^{3}\right.$, dd, $J$ $=8.0,2.0 \mathrm{~Hz}, 1 \mathrm{H}), 8.06\left(\mathrm{H}^{8}, \mathrm{dd}, J=8.0,1.2 \mathrm{~Hz}, 1 \mathrm{H}\right), 7.97\left(\mathrm{H}^{4}, \mathrm{~d}, J=8.8,1 \mathrm{H}\right), 7.85\left(\mathrm{H}^{5}, \mathrm{~d}, J=\right.$ $8.8 \mathrm{~Hz}, 1 \mathrm{H}), 7.73\left(\mathrm{H}^{7}, \mathrm{t}, J=8.0 \mathrm{~Hz}, 1 \mathrm{H}\right), 7.50\left(\mathrm{H}^{6}, \mathrm{dd}, J=7.2,1.6 \mathrm{~Hz}, 1 \mathrm{H}\right), 7.46\left(\mathrm{H}^{2}, \mathrm{dd}, J=8.0\right.$, $4.0 \mathrm{~Hz}, 1 \mathrm{H}), 7.29\left(\mathrm{H}^{9}, \mathrm{dt}, J=10.8,8.4 \mathrm{~Hz}, 1 \mathrm{H}\right), 7.19\left(\mathrm{H}^{11}\right.$, ddd, $\left.J=11.8,8.4,2.0 \mathrm{~Hz}, 1 \mathrm{H}\right), 7.05$ $\left(\mathrm{H}^{10}, \mathrm{~m}, 1 \mathrm{H}\right) ;{ }^{13} \mathrm{C} \mathrm{NMR}\left(100 \mathrm{MHz}\right.$, acetone- $\left.d_{6}\right): \delta 150.36(\mathrm{dd}, J=242.1,12.8 \mathrm{~Hz}), 149.65(\mathrm{dd}, J$ $=241.7,12.5 \mathrm{~Hz}), 147.91,147.18,144.82(\mathrm{dd}, J=6.4,3.6 \mathrm{~Hz}), 140.25(\mathrm{~d}, J=1.4 \mathrm{~Hz}), 136.54$, 136.05, 132.03, 129.68, 129.63, 129.14, 128.41, 128.06, 127.27, 125.98 (dd, $J=5.9,3.7 \mathrm{~Hz}$ ), 122.49, $118.58(\mathrm{~d}, J=16.8 \mathrm{~Hz}), 116.87(\mathrm{~d}, J=16.8 \mathrm{~Hz}) ;{ }^{19} \mathrm{~F}$ NMR (acetone- $\left.d_{6}\right): \delta-142.50(\mathrm{~m}$, 
1F), $-145.13(\mathrm{~m}, 1 \mathrm{~F})$. HRMS ESI with Formic Acid (m/z): $[\mathrm{M}+\mathrm{H}]^{+}$calcd for $\mathrm{C}_{19} \mathrm{H}_{12} \mathrm{NF}_{2}$, 292.0938; found, 292.0930.

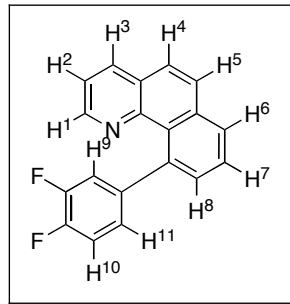

\section{Confirmation of regioselectivity (assignments based on GCOSY, HSQC, and coupling constant analysis)}
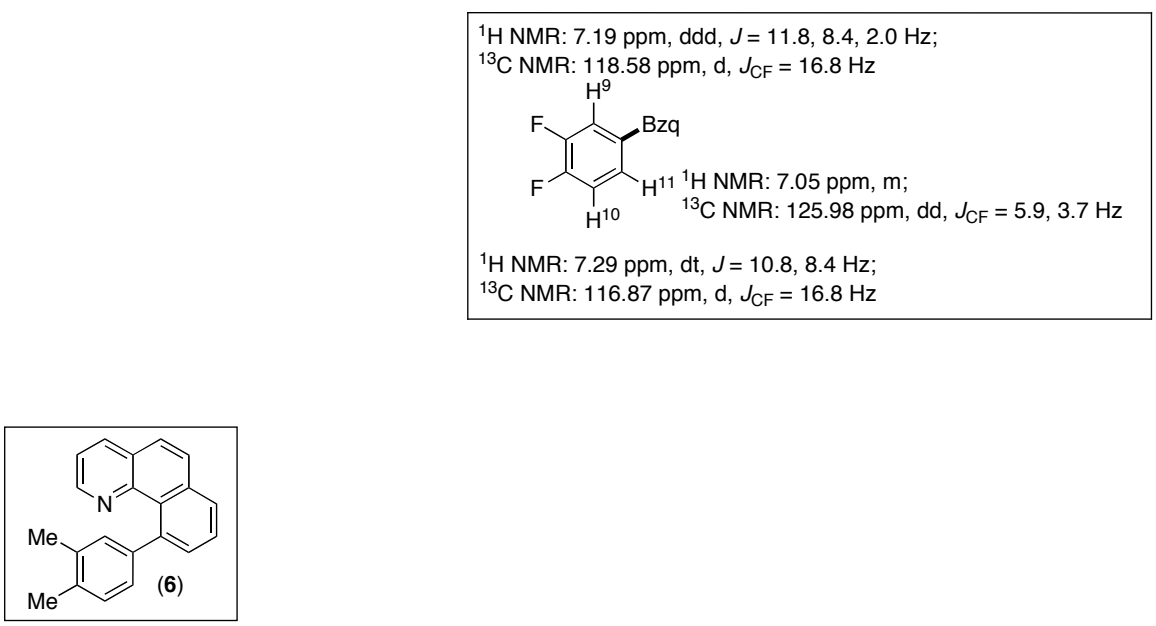

Product 6. $\mathrm{L} \sim \mathrm{C}_{\mathrm{Ar}}-\mathrm{H}=7,8$-benzo[h]quinoline; $\mathrm{Ar}-\mathrm{H}=o$-xylene; temperature $=130{ }^{\circ} \mathrm{C}$. The product was isolated as an off-white solid $\left(81 \mathrm{mg}, 67 \%\right.$ yield, $\mathrm{mp}=111.5-114.6{ }^{\circ} \mathrm{C}, \mathrm{rf}=0.11 \mathrm{in}$ $98 \%$ hexanes $/ 2 \%$ EtOAc). ${ }^{1} \mathrm{H}$ NMR $\left(500 \mathrm{MHz}\right.$, acetone- $\left.d_{6}\right): \delta 8.39\left(\mathrm{H}^{1}, \mathrm{dd}, J=3.4,1.4 \mathrm{~Hz}, 1 \mathrm{H}\right)$, $8.24\left(\mathrm{H}^{3}, \mathrm{dd}, J=6.4,1.6 \mathrm{~Hz}, 1 \mathrm{H}\right), 7.99\left(\mathrm{H}^{8}, \mathrm{dd}, J=6.2,1.0 \mathrm{~Hz}, 1 \mathrm{H}\right), 7.94\left(\mathrm{H}^{4}, \mathrm{~d}, J=7.2 \mathrm{~Hz}\right.$, $1 \mathrm{H}), 7.81\left(\mathrm{H}^{5}, \mathrm{~d}, J=7.2 \mathrm{~Hz}, 1 \mathrm{H}\right), 7.70\left(\mathrm{H}^{7}, \mathrm{t}, J=6.0 \mathrm{~Hz}, 1 \mathrm{H}\right), 7.48\left(\mathrm{H}^{6}, \mathrm{dd}, J=5.6,1.2 \mathrm{~Hz}, 1 \mathrm{H}\right)$, $7.41\left(\mathrm{H}^{2}, \mathrm{dd}, J=6.4,3.2 \mathrm{~Hz}, 1 \mathrm{H}\right), 7.10\left(\mathrm{H}^{10}, \mathrm{~d}, J=6.0 \mathrm{~Hz}, 1 \mathrm{H}\right), 7.08\left(\mathrm{H}^{13}, \mathrm{bs}, 1 \mathrm{H}\right), 7.00\left(\mathrm{H}^{9}, \mathrm{dd}\right.$, $J=6.0,1.2 \mathrm{~Hz}, 1 \mathrm{H}), 2.32\left(\mathrm{H}^{11}\right.$ or $\left.\mathrm{H}^{12}, \mathrm{~s}, 3 \mathrm{H}\right), 2.26\left(\mathrm{H}^{11}\right.$ or $\left.\mathrm{H}^{12}, \mathrm{~s}, 3 \mathrm{H}\right) ;{ }^{13} \mathrm{C} \mathrm{NMR}(125 \mathrm{MHz}$, actone- $\left.d_{6}\right): \delta 147.72,147.61,145.04,142.94,136.26,136.13,135.70,134.09,132.49,130.80$, $129.87,129.35,129.23,128.77,128.25,127.98,127.23,126.97,122.24,19.99,19.66$. HRMS ESI with Formic Acid (m/z): $[\mathrm{M}+\mathrm{H}]^{+}$calcd for $\mathrm{C}_{21} \mathrm{H}_{17} \mathrm{~N}, 284.1439$; found, 284.1426. 


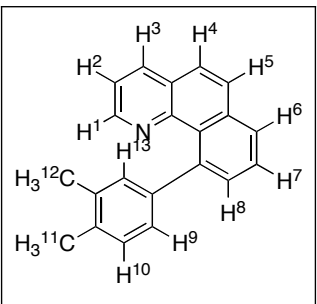

\section{Confirmation of regioselectivity (assignments based on COSY analysis)}
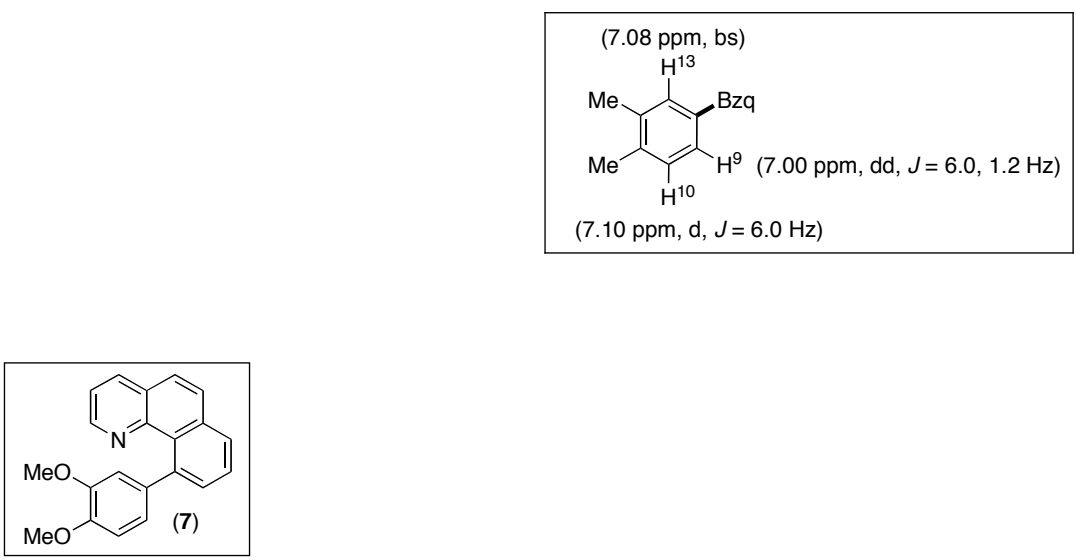

Product 7. $\mathrm{L} \sim \mathrm{C}_{\mathrm{Ar}}-\mathrm{H}=7,8$-benzo[ $\left.h\right]$ quinoline; $\mathrm{Ar}-\mathrm{H}=o$-dimethoxybenzene; temperature $=130$ ${ }^{\circ} \mathrm{C}$. The product was isolated as a off-white crystalline solid $(88 \mathrm{mg}, 66 \%$ yield, $\mathrm{mp}=133.6-$ $134.4{ }^{\circ} \mathrm{C}$, $\mathrm{rf}=0.11$ in $90 \%$ hexanes $/ 10 \%$ EtOAc). Trace amounts $(<5 \%)$ of the Bzq-Bzq dimer were observed in the ${ }^{1} \mathrm{H}$ NMR spectrum of the crude reaction mixture. ${ }^{1} \mathrm{H}$ NMR (400 MHz, acetone- $\left.d_{6}\right): \delta 8.46\left(\mathrm{H}^{1}, \mathrm{dd}, J=4.2,1.8 \mathrm{~Hz}, 1 \mathrm{H}\right), 8.27\left(\mathrm{H}^{3}, \mathrm{dd}, J=8.0,2.0 \mathrm{~Hz}, 1 \mathrm{H}\right), 8.01\left(\mathrm{H}^{8}, \mathrm{dd}\right.$, $J=7.8,1.4 \mathrm{~Hz}, 1 \mathrm{H}), 7.96\left(\mathrm{H}^{4}, \mathrm{~d}, J=8.8 \mathrm{~Hz}, 1 \mathrm{H}\right), 7.83\left(\mathrm{H}^{5}, \mathrm{~d}, J=8.8 \mathrm{~Hz}, 1 \mathrm{H}\right), 7.71\left(\mathrm{H}^{7}, \mathrm{t}, J=\right.$ $7.6 \mathrm{~Hz}, 1 \mathrm{H}), 7.53\left(\mathrm{H}^{6}, \mathrm{dd}, J=7.2,1.2 \mathrm{~Hz}, 1 \mathrm{H}\right), 7.44\left(\mathrm{H}^{2}, \mathrm{dd}, J=8.0,4.4 \mathrm{~Hz}, 1 \mathrm{H}\right), 6.95\left(\mathrm{H}^{10}, \mathrm{~d}, J\right.$ $=8.0 \mathrm{~Hz}, 1 \mathrm{H}), 6.88\left(\mathrm{H}^{13}, \mathrm{~d}, J=2.0 \mathrm{~Hz}, 1 \mathrm{H}\right), 6.82\left(\mathrm{H}^{9}, \mathrm{dd}, J=8.0,2.0 \mathrm{~Hz}, 1 \mathrm{H}\right), 3.87\left(\mathrm{H}^{11}\right.$ or H $\mathrm{H}^{12}$, s, 3H), $3.70\left(\mathrm{H}^{11}\right.$ or $\mathrm{H}^{12}$, s, $\left.3 \mathrm{H}\right) ;{ }^{13} \mathrm{C}$ NMR (100 MHz, acetone- $\left.d_{6}\right): \delta 149.52,148.94,147.72$, $147.71,142.66,140.31,136.32,136.18,132.51,129.94,129.24,128.84,128.31,128.00,127.03$, 122.29, 121.59, 114.89, 112.20, 56.25, 56.20. HRMS ESI with Formic Acid (m/z): $[\mathrm{M}+\mathrm{H}]^{+}$calcd for $\mathrm{C}_{21} \mathrm{H}_{17} \mathrm{O}_{2} \mathrm{~N}, 316.1338$; found, 316.1324 .

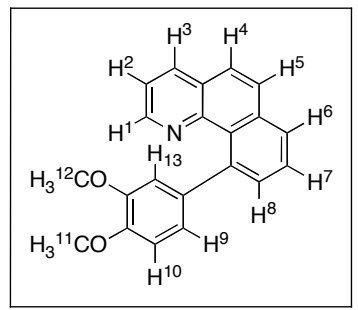




\section{Confirmation of regioselectivity (assignments based on COSY analysis)}
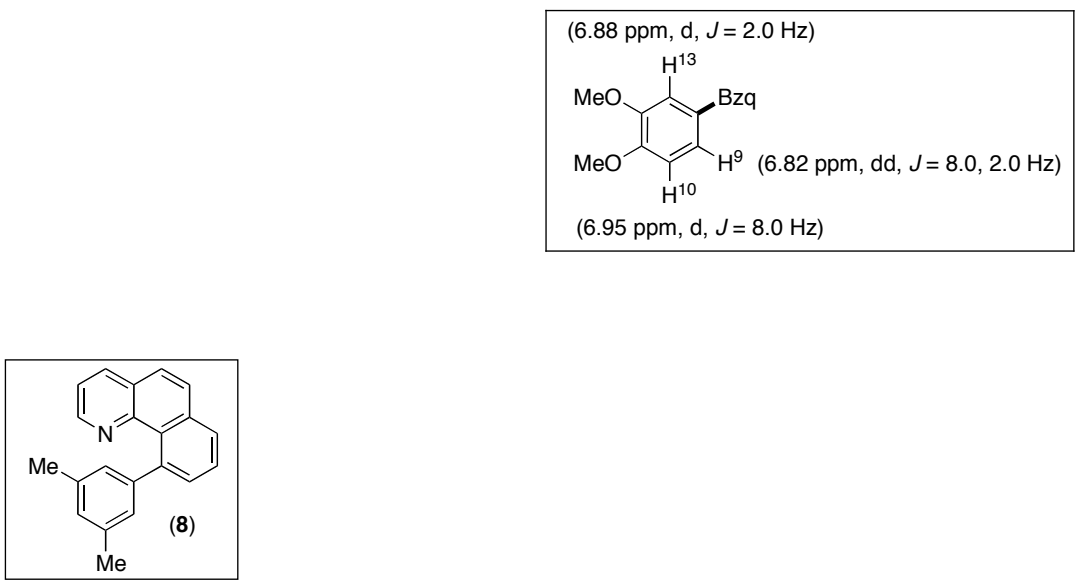

Product 8. $\mathrm{L} \sim \mathrm{C}_{\mathrm{Ar}}-\mathrm{H}=7,8$-benzo $[h]$ quinoline; $\mathrm{Ar}-\mathrm{H}=m$-xylene; temperature $=150{ }^{\circ} \mathrm{C}$. The product was isolated as a pale orange viscous oil $(84 \mathrm{mg}, 69 \%$ yield, $\mathrm{rf}=0.11$ in $98 \%$ hexanes $/ 2 \%$ EtOAc). Trace amounts $(<5 \%)$ of the Bzq-Bzq dimer were observed in the ${ }^{1} \mathrm{H}$ NMR spectrum of the crude reaction mixture. ${ }^{1} \mathrm{H}$ NMR $\left(400 \mathrm{MHz}\right.$, acetone- $\left.d_{6}\right): \delta 8.41\left(\mathrm{H}^{1}, \mathrm{dd}, J=4.4\right.$, $2.0 \mathrm{~Hz}, 1 \mathrm{H}), 8.25\left(\mathrm{H}^{3}, \mathrm{dd}, J=8.0,2.0 \mathrm{~Hz}, 1 \mathrm{H}\right), 8.00\left(\mathrm{H}^{8}, \mathrm{dd}, J=8.0,1.2 \mathrm{~Hz}, 1 \mathrm{H}\right), 7.96\left(\mathrm{H}^{4}, \mathrm{~d}, J\right.$ $=8.8 \mathrm{~Hz}, 1 \mathrm{H}), 7.82\left(\mathrm{H}^{5}, \mathrm{~d}, J=8.8 \mathrm{~Hz}, 1 \mathrm{H}\right), 7.70\left(\mathrm{H}^{7}, \mathrm{t}, J=7.6 \mathrm{~Hz}, 1 \mathrm{H}\right), 7.48\left(\mathrm{H}^{6}, \mathrm{dd}, J=7.6,1.2\right.$ $\mathrm{Hz}, 1 \mathrm{H}), 7.42\left(\mathrm{H}^{2}, \mathrm{dd}, J=8.0,4.0 \mathrm{~Hz}, 1 \mathrm{H}\right), 6.96\left(\mathrm{H}^{11}, \mathrm{~s}, 1 \mathrm{H}\right), 6.90\left(\mathrm{H}^{9}, \mathrm{~s}, 2 \mathrm{H}\right), 2.30\left(\mathrm{H}^{10}, \mathrm{~s}, 6 \mathrm{H}\right)$; ${ }^{13} \mathrm{C}$ NMR $\left(100 \mathrm{MHz}, \mathrm{CDCl}_{3}\right): \delta 146.82,146.08,141.88,136.58,135.10,134.98,131.52,129.57$, $128.98,128.29,127.72,127.31,127.15,126.98,126.55,125.79,121.00,21.45$. HRMS ESI with Formic Acid (m/z): $[\mathrm{M}+\mathrm{H}]^{+}$calcd for $\mathrm{C}_{21} \mathrm{H}_{18} \mathrm{~N}, 284.1439$; found, 284.1429.

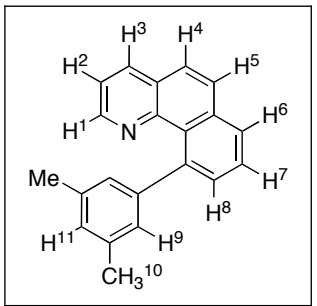

Confirmation of regioselectivity

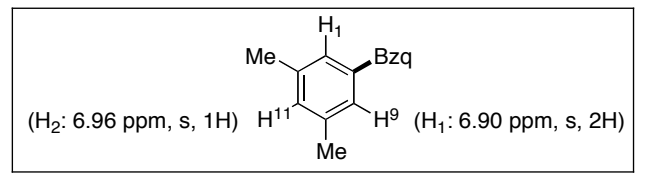




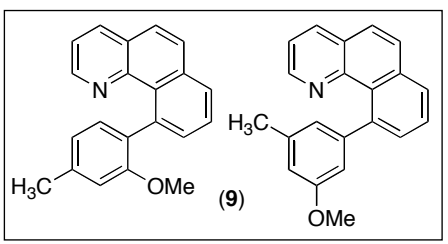

Product 9. $\mathrm{L} \sim \mathrm{C}_{\mathrm{Ar}}-\mathrm{H}=7,8$-benzo[ $\left.h\right]$ quinoline; $\mathrm{Ar}-\mathrm{H}=3$-methylanisole; temperature $=150{ }^{\circ} \mathrm{C}$. The products were isolated as a mixture of isomers, a pale yellow oil ( $\mathrm{rf}=0.26$ in $95 \%$ hexanes $/ 5 \%$ EtOAc, $90.0 \mathrm{mg}, 70 \%$ yield, $2.5: 1$ ratio of the 1,3,5:1,3,4 isomers). Trace amounts $(<5 \%)$ of the Bzq-Bzq dimer were observed in the ${ }^{1} \mathrm{H}$ NMR spectrum of the crude reaction mixture. Isomers were separated by HPLC to afford each isomer as pale yellow oils.

1,3,5 isomer. ${ }^{1} \mathrm{H}$ NMR (400 MHz, acetone- $\left.d_{6}\right): \delta 8.44\left(\mathrm{H}^{1}, \mathrm{dd}, J=4.4,2.0 \mathrm{~Hz}, 1 \mathrm{H}\right), 8.26\left(\mathrm{H}^{3}, \mathrm{dd}\right.$, $J=8.0,2.0 \mathrm{~Hz}, 1 \mathrm{H}), 8.02\left(\mathrm{H}^{8}, \mathrm{dd}, J=7.8,1.4 \mathrm{~Hz}, 1 \mathrm{H}\right), 7.96\left(\mathrm{H}^{4}, \mathrm{~d}, J=8.8 \mathrm{~Hz}, 1 \mathrm{H}\right), 7.83\left(\mathrm{H}^{5}, \mathrm{~d}\right.$, $J=8.8 \mathrm{~Hz}, 1 \mathrm{H}), 7.71\left(\mathrm{H}^{7}, \mathrm{t}, J=7.6 \mathrm{~Hz}, 1 \mathrm{H}\right), 7.51\left(\mathrm{H}^{6}, \mathrm{dd}, J=7.2,1.2 \mathrm{~Hz}, 1 \mathrm{H}\right), 7.43\left(\mathrm{H}^{2}, \mathrm{dd}, J=\right.$ 8.0, $4.4 \mathrm{~Hz}, 1 \mathrm{H}), 6.73\left(\mathrm{H}^{9}\right.$ or $\mathrm{H}^{11}$ or $\mathrm{H}^{13}$, bs, $\left.1 \mathrm{H}\right), 6.69\left(\mathrm{H}^{9}\right.$ or $\mathrm{H}^{11}$ or $\mathrm{H}^{13}$, bs, $\left.1 \mathrm{H}\right), 6.67\left(\mathrm{H}^{9}\right.$ or $\mathrm{H}^{11}$ or $\mathrm{H}^{13}$, bs, $\left.1 \mathrm{H}\right), 3.74\left(\mathrm{H}^{12}, \mathrm{~s}, 3 \mathrm{H}\right), 2.32\left(\mathrm{H}^{10}, \mathrm{~s}, 3 \mathrm{H}\right) ;{ }^{13} \mathrm{C}$ NMR (100 MHz, acetone- $\left.d_{6}\right): \delta 160.14$, 148.60, 147.77, 147.56, 142.76, 138.55, 136.27, 136.06, 132.09, 129.82, 129.19, 128.96, 128.29, 127.97, 127.04, 122.93, 122.30, 112.96, 112.44, 55.41, 21.78. HRMS ESI with Formic Acid $(\mathrm{m} / \mathrm{z}):[\mathrm{M}+\mathrm{H}]^{+}$calcd for $\mathrm{C}_{21} \mathrm{H}_{18} \mathrm{NO}, 300.1388$; found, 300.1382 .

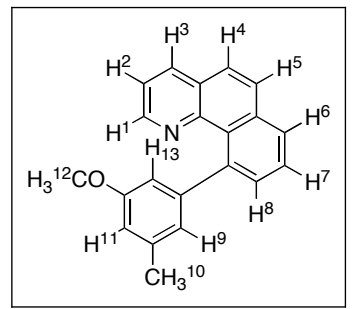

\section{Confirmation of Regioselectivity (assignments based on COSY and HSQC)}

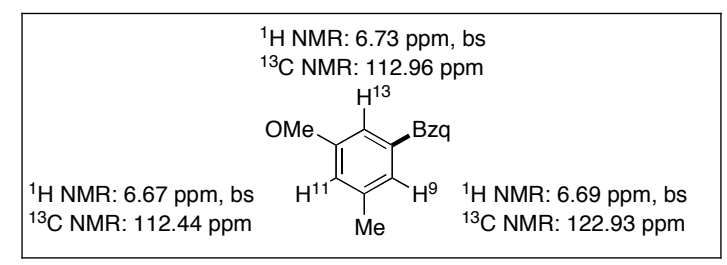

1,2,4 isomer. Regiochemistry confirmed by GCOSY and HSQC. ${ }^{1} \mathrm{H}$ NMR (400 MHz, acetone$\left.d_{6}\right): \delta 8.42\left(\mathrm{H}^{1}, \mathrm{dd}, J=4.2,1.8 \mathrm{~Hz}, 1 \mathrm{H}\right), 8.24\left(\mathrm{H}^{3}, \mathrm{dd}, J=8.0,2.0 \mathrm{~Hz}, 1 \mathrm{H}\right), 7.99\left(\mathrm{H}^{8}, \mathrm{dd}, J=7.8\right.$, $1.0 \mathrm{~Hz}, 1 \mathrm{H}), 7.94\left(\mathrm{H}^{4}, \mathrm{~d}, J=8.8 \mathrm{~Hz}, 1 \mathrm{H}\right), 7.80\left(\mathrm{H}^{5}, \mathrm{~d}, J=8.8 \mathrm{~Hz}, 1 \mathrm{H}\right), 7.71\left(\mathrm{H}^{7}, \mathrm{t}, J=7.4 \mathrm{~Hz}\right.$, 
1H), $7.45\left(\mathrm{H}^{6}, \mathrm{dd}, J=7.4,1.0 \mathrm{~Hz}, 1 \mathrm{H}\right), 7.41\left(\mathrm{H}^{2}, \mathrm{dd}, J=8.0,4.0 \mathrm{~Hz}, 1 \mathrm{H}\right), 7.04\left(\mathrm{H}^{12}, \mathrm{~d}, J=7.2\right.$ $\mathrm{Hz}, 1 \mathrm{H}), 6.84-6.78\left(\mathrm{H}^{10}\right.$ and $\mathrm{H}^{13}$, multiple peaks, $\left.2 \mathrm{H}\right), 3.36\left(\mathrm{H}^{9}, \mathrm{~s}, 3 \mathrm{H}\right), 2.43\left(\mathrm{H}^{11}, \mathrm{~s}, 3 \mathrm{H}\right) ;{ }^{13} \mathrm{C}$ NMR (100 MHz, acetone- $\left.d_{6}\right)$ : $\delta 158.43,148.20,147.81,139.44,137.68,136.07,135.64,134.21$, 132.36, 130.89, 129.72, 129.31, 128.82, 128.08, 127.81, 126.74, 122.04, 121.34, 111.64, 55.31, 21.81. HRMS ESI with Formic Acid $(\mathrm{m} / \mathrm{z}):[\mathrm{M}+\mathrm{H}]^{+}$calcd for $\mathrm{C}_{21} \mathrm{H}_{18} \mathrm{NO}, 300.1388$; found, 300.1393 .

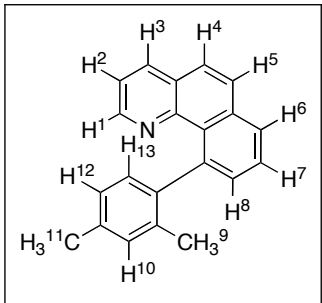

\section{Confirmation of regioselectivity (assignments based on GCOSY and HSQC)}

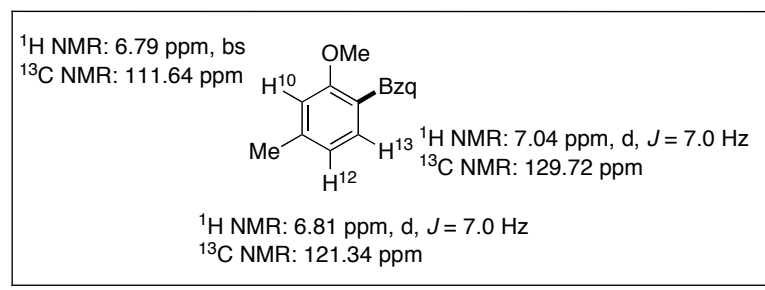

The assignment of the benzoquinoline coupled ortho to the OMe rather than the Me is based on the ${ }^{13} \mathrm{C}$ NMR chemical shifts of the $\mathbf{C}-\mathrm{H}_{2}$ and $\mathbf{C}-\mathrm{H}_{3}$. As seen 1,3,5-isomer (above), a $\mathbf{C}_{\mathbf{A r}}-\mathbf{H}$ ortho to a $\mathrm{C}_{\mathrm{Ar}}-\mathrm{OMe}$ is expected to appear at between $110-115 \mathrm{ppm}$ in the ${ }^{13} \mathrm{C}$ NMR. Only one $\mathrm{C}_{\mathrm{Ar}}-\mathrm{H}$ appears in this region of the ${ }^{13} \mathrm{C}$ NMR spectrum of this compound, and this is identified as $\mathbf{C}-\mathrm{H}_{1}$.

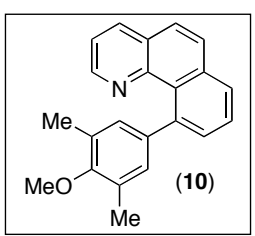

Product 10. $\mathrm{L} \sim \mathrm{C}_{\mathrm{Ar}}-\mathrm{H}=7,8$-benzo[ $\left.h\right]$ quinoline; $\mathrm{Ar}-\mathrm{H}=2$,6-dimethylanisole; temperature $=150$ ${ }^{\circ} \mathrm{C}$. The product was isolated as a pale orange viscous oil $(89 \mathrm{mg}, 66 \%$ yield, $\mathrm{rf}=0.14$ in $95 \%$ hexanes $/ 5 \%$ EtOAc). Trace amounts $(<5 \%)$ of the Bzq-Bzq dimer were observed in the ${ }^{1} \mathrm{H}$ NMR spectrum of the crude reaction mixture. ${ }^{1} \mathrm{H}$ NMR $\left(400 \mathrm{MHz}\right.$, acetone- $\left.d_{6}\right): \delta 8.42\left(\mathrm{H}^{1}, \mathrm{dd}, J=4.0\right.$, $2.0 \mathrm{~Hz}, 1 \mathrm{H}), 8.24\left(\mathrm{H}^{3}\right.$, dd $\left., J=8.0,2.0 \mathrm{~Hz}, 1 \mathrm{H}\right), 7.98\left(\mathrm{H}^{8}, \mathrm{dd}, J=8.0,1.6 \mathrm{~Hz}, 1 \mathrm{H}\right), 7.94\left(\mathrm{H}^{4}, \mathrm{~d}, J=\right.$ 
$8.8 \mathrm{~Hz}, 1 \mathrm{H}), 7.81\left(\mathrm{H}^{5}, \mathrm{~d}, J=8.8 \mathrm{~Hz}, 1 \mathrm{H}\right), 7.69\left(\mathrm{H}^{7}, \mathrm{dd}, J=8.0,7.2 \mathrm{~Hz}, 1 \mathrm{H}\right), 7.49\left(\mathrm{H}^{6}, \mathrm{dd}, J=\right.$ 7.2, $1.6 \mathrm{~Hz}, 1 \mathrm{H}), 7.41\left(\mathrm{H}^{2}, \mathrm{dd}, J=8.0,4.0 \mathrm{~Hz}, 1 \mathrm{H}\right), 6.93\left(\mathrm{H}^{9}, \mathrm{bs}, 2 \mathrm{H}\right), 3.78\left(\mathrm{H}^{11}, \mathrm{~s}, 3 \mathrm{H}\right), 2.27$ $\left(\mathrm{H}^{10}, \mathrm{~s}, 6 \mathrm{H}\right) ;{ }^{13} \mathrm{C} \mathrm{NMR}\left(100 \mathrm{MHz}\right.$, acetone- $\left.d_{6}\right): \delta 149.52,148.94,142.65,140.31,136.31$, $136.19,132.51,129.94,129.24,128.84,128.30,128.00,127.03,122.29,121.59,114.89,112.20$, 55.42, 21.78. HRMS ESI with Formic Acid $(\mathrm{m} / \mathrm{z})$ : $[\mathrm{M}+\mathrm{H}]^{+}$calcd for $\mathrm{C}_{22} \mathrm{H}_{20} \mathrm{NO}, 314.1545$; found, 314.1544 .

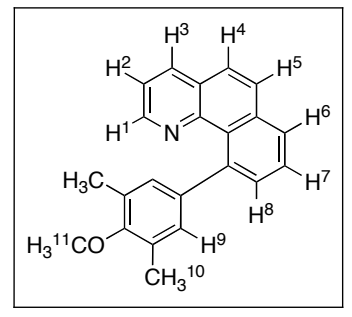

\section{Confirmation of Regioselectivity}

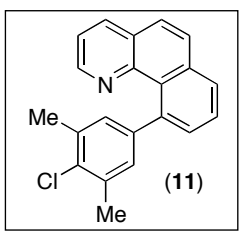

Product 11. $\mathrm{L} \sim \mathrm{C}_{\mathrm{Ar}}-\mathrm{H}=7,8$-benzo $[h]$ quinoline; $\mathrm{Ar}-\mathrm{H}=2$-chloro- $m$-xylene; temperature $=150$ ${ }^{\circ} \mathrm{C}$. The product was isolated as off-white solid $\left(101 \mathrm{mg}, 74 \%\right.$ yield, $\mathrm{mp}=118.0-121.7^{\circ} \mathrm{C}, \mathrm{rf}=$ 0.19 in $98 \%$ hexanes $/ 2 \%$ EtOAc). ${ }^{1} \mathrm{H}$ NMR (400 MHz, acetone- $\left.d_{6}\right): \delta 8.42\left(\mathrm{H}^{1}, \mathrm{dd}, J=4.4,2.0\right.$ $\mathrm{Hz}, 1 \mathrm{H}), 8.26\left(\mathrm{H}^{3}, \mathrm{dd}, J=8.0,2.0 \mathrm{~Hz}, 1 \mathrm{H}\right), 8.02\left(\mathrm{H}^{8}, \mathrm{dd}, J=8.0,1.2 \mathrm{~Hz}, 1 \mathrm{H}\right), 7.96\left(\mathrm{H}^{4}, \mathrm{~d}, J=\right.$ $8.8 \mathrm{~Hz}, 1 \mathrm{H}), 7.83\left(\mathrm{H}^{5}, \mathrm{~d}, J=8.8 \mathrm{~Hz}, 1 \mathrm{H}\right), 7.71\left(\mathrm{H}^{7}, \mathrm{t}, J=7.6 \mathrm{~Hz}, 1 \mathrm{H}\right), 7.47\left(\mathrm{H}^{6}, \mathrm{dd}, J=7.2,1.2\right.$ $\mathrm{Hz}, 1 \mathrm{H}), 7.43\left(\mathrm{H}^{2}, \mathrm{dd}, J=8.0,3.6 \mathrm{~Hz}, 1 \mathrm{H}\right), 7.07\left(\mathrm{H}^{9}, \mathrm{~s}, 2 \mathrm{H}\right), 2.37\left(\mathrm{H}^{10}, \mathrm{~s}, 6 \mathrm{H}\right) ;{ }^{13} \mathrm{C} \mathrm{NMR}(100$ $\left.\mathrm{MHz}, \mathrm{CDCl}_{3}\right): \delta 146.86,146.66,144.08,140.67,135.20,134.97,134.81,132.06,131.44$, 128.84, 128.81, 128.25, 128.02, 127.18, 126.98, 125.91, 121.11, 20.78. HRMS ESI with Formic Acid (m/z): $[\mathrm{M}+\mathrm{H}]^{+}$calcd for $\mathrm{C}_{21} \mathrm{H}_{17} \mathrm{NCl}, 318.1050$; found, 318.1042 . 


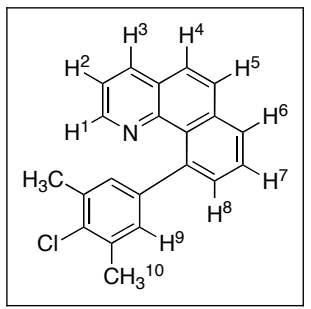

\section{Confirmation of Regioselectivity}
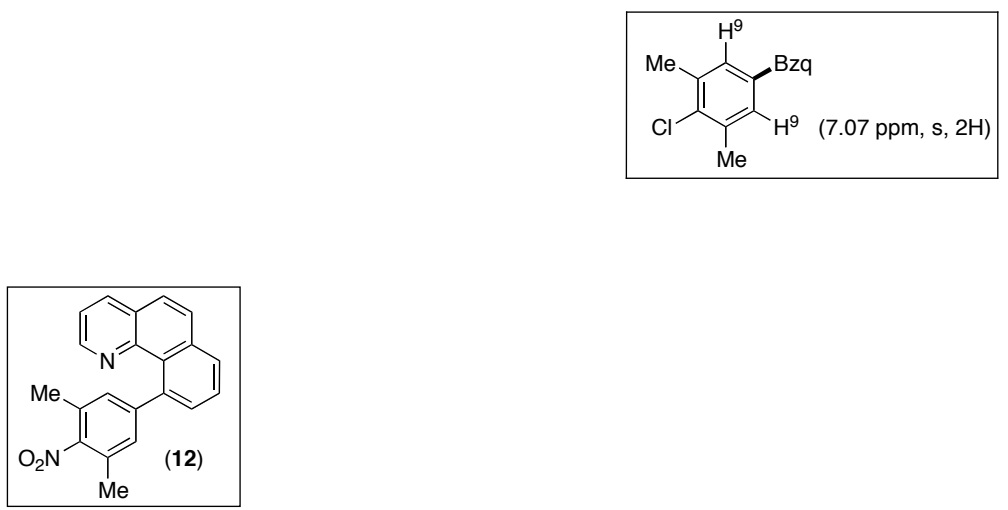

Product 12. $\mathrm{L} \sim \mathrm{C}_{\mathrm{Ar}}-\mathrm{H}=7,8$-benzo $[h]$ quinoline; $\mathrm{Ar}-\mathrm{H}=2$-nitro- $m$-xylene; temperature $=150{ }^{\circ} \mathrm{C}$. GC analysis of the crude reaction mixture showed an $\sim 20: 1$ ratio of regioisomers. The major isomer was isolated as white solid and was purified from the 1,2,3,4 isomer by HPLC (79 mg, $56 \%$ yield, $\mathrm{rf}=0.17$ in $95 \%$ hexanes $/ 5 \%$ EtOAc). Trace amounts $(<5 \%)$ of the Bzq-Bzq dimer were observed in the ${ }^{1} \mathrm{H}$ NMR spectrum of the crude reaction mixture. By GC the a peak which may correspond to the $1,2,3,4$ isomer is present, in a $>18: 1$ ratio. ${ }^{1} \mathrm{H}$ NMR (400 $\mathrm{MHz}$, acetone$\left.d_{6}\right): \delta 8.46\left(\mathrm{H}^{1}, \mathrm{dd}, J=4.2,1.8 \mathrm{~Hz}, 1 \mathrm{H}\right), 8.32\left(\mathrm{H}^{3}, \mathrm{dd}, J=8.0,2.0 \mathrm{~Hz}, 1 \mathrm{H}\right), 8.10\left(\mathrm{H}^{8}, \mathrm{dd}, J=8.0\right.$, $1.2 \mathrm{~Hz}, 1 \mathrm{H}), 8.01\left(\mathrm{H}^{4}, \mathrm{~d}, J=8.8 \mathrm{~Hz}, 1 \mathrm{H}\right), 7.89\left(\mathrm{H}^{5}, \mathrm{~d}, J=8.8 \mathrm{~Hz}, 1 \mathrm{H}\right), 7.77\left(\mathrm{H}^{7}, \mathrm{t}, J=7.6 \mathrm{~Hz}\right.$, $1 \mathrm{H}), 7.52\left(\mathrm{H}^{6}, \mathrm{dd}, J=7.4,1.0 \mathrm{~Hz}, 1 \mathrm{H}\right), 7.49\left(\mathrm{H}^{2}, \mathrm{dd}, J=7.8,4.2 \mathrm{~Hz}, 1 \mathrm{H}\right), 7.16\left(\mathrm{H}^{9}, \mathrm{~s}, 2 \mathrm{H}\right), 2.33$ $\left(\mathrm{H}^{10}, \mathrm{~s}, 6 \mathrm{H}\right) ;{ }^{13} \mathrm{C} \mathrm{NMR}\left(100 \mathrm{MHz}\right.$, acetone- $\left.d_{6}\right): \delta 149.74,147.89,147.13,140.67,136.58$, $136.08,132.00,130.14,129.71,129.51,129.18,129.17,128.43,128.14,127.29,122.62,17.65$ (two carbons are coincidentally overlapping). HRMS ESI with Formic Acid (m/z): $[\mathrm{M}+\mathrm{H}]^{+}$calcd for $\mathrm{C}_{21} \mathrm{H}_{16} \mathrm{~N}_{2} \mathrm{O}_{2} 329.1279$; found, 329.1290.

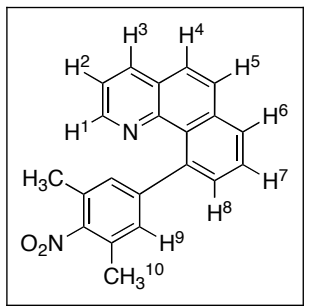




\section{Confirmation of Regioselectivity}
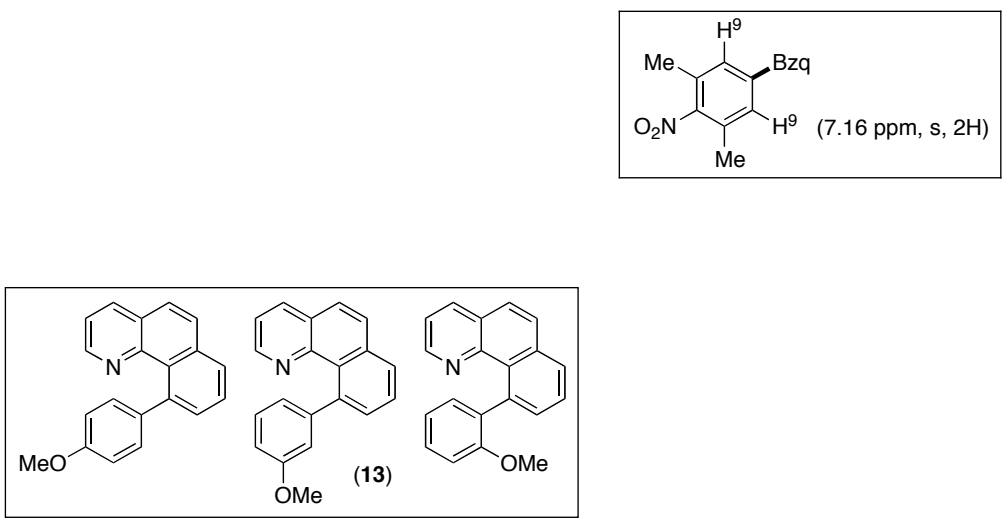

Product 13: $\mathrm{L} \sim \mathrm{C}_{\mathrm{Ar}}-\mathrm{H}=7,8$-benzo $[h]$ quinoline; $\mathrm{Ar}-\mathrm{H}=$ anisole; temperature $=130{ }^{\circ} \mathrm{C}$. The product were isolated as a mixture of isomers as a pale orange viscous oil ( $\mathrm{rf}=0.13$ in $95 \%$ hexanes $/ 5 \%$ EtOAc, $99 \mathrm{mg}, 80 \%$ yield, the $o: m: p$ ratio of $1: 2.6: 3.3$ was determined by GC analysis of crude reaction mixture). Trace amounts $(<5 \%)$ of the Bzq-Bzq dimer were observed in the ${ }^{1} \mathrm{H}$ NMR spectrum of the crude reaction mixture. The three regioisomers were separated by HPLC.

ortho isomer: ${ }^{1} \mathrm{H}$ NMR (400 MHz, acetone- $\left.d_{6}\right)$ : $\delta 8.40\left(\mathrm{H}^{1}, \mathrm{dd}, J=4.4,2.0 \mathrm{~Hz}, 1 \mathrm{H}\right), 8.26\left(\mathrm{H}^{3}\right.$, dd, $J=8.0,2.0 \mathrm{~Hz}, 1 \mathrm{H}), 8.02\left(\mathrm{H}^{8}, \mathrm{dd}, J=8.2,1.4 \mathrm{~Hz}, 1 \mathrm{H}\right), 7.96\left(\mathrm{H}^{4}, \mathrm{~d}, J=8.8 \mathrm{~Hz}, 1 \mathrm{H}\right), 7.82\left(\mathrm{H}^{5}, \mathrm{~d}\right.$, $J=8.8 \mathrm{~Hz}, 1 \mathrm{H}), 7.73\left(\mathrm{H}^{7}, \mathrm{t}, J=7.6 \mathrm{~Hz}, 1 \mathrm{H}\right), 7.47\left(\mathrm{H}^{6}, \mathrm{dd}, J=7.2,1.2 \mathrm{~Hz}, 1 \mathrm{H}\right), 7.42\left(\mathrm{H}^{2} . \mathrm{dd}, J=\right.$ 8.0, 4.0 Hz, 1H), $7.33\left(\mathrm{H}^{11}\right.$, ddd, $\left.J=8.0,7.6,2.0 \mathrm{~Hz}, 1 \mathrm{H}\right), 7.16\left(\mathrm{H}^{9}, \mathrm{dd}, J=7.4,2.0 \mathrm{~Hz}, 1 \mathrm{H}\right)$, $6.99\left(\mathrm{H}^{10}\right.$, app. $\left.\mathrm{td}, J=7.4,0.8 \mathrm{~Hz}, 1 \mathrm{H}\right), 6.96\left(\mathrm{H}^{12}, \mathrm{dd}, J=8.0,0.8 \mathrm{~Hz}, 1 \mathrm{H}\right), 3.39\left(\mathrm{H}^{13}, \mathrm{~s}, 3 \mathrm{H}\right) ;{ }^{13} \mathrm{C}$ NMR (100 MHz, acetone- $\left.d_{6}\right): \delta 158.56,148.07,147.88,139.34,137.04,136.11,135.62,132.15$, $130.76,129.89,129.30,128.96,128.24,128.12,127.83,126.79,122.11,120.83,110.64,55.37$. HRMS ESI with Formic Acid (m/z): $[\mathrm{M}+\mathrm{H}]^{+}$calcd for $\mathrm{C}_{20} \mathrm{H}_{16} \mathrm{NO}, 286.1232$; found, 286.1219.

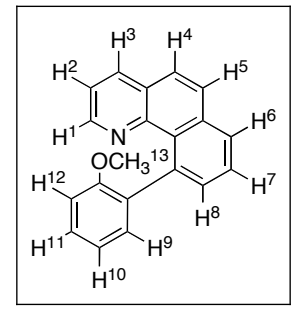

Confirmation of Regioselectivity (assignments based on COSY and HSQC) 


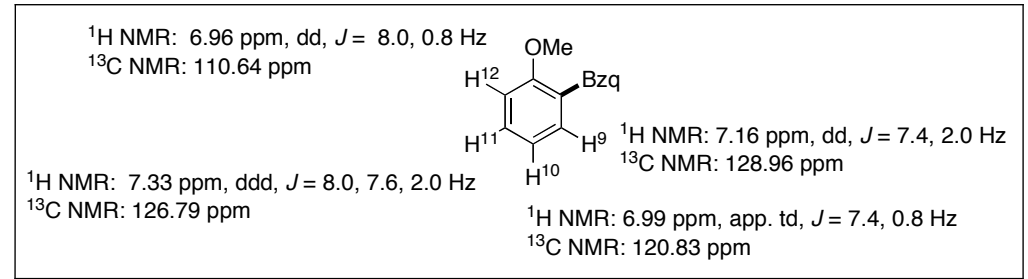

meta isomer: ${ }^{1} \mathrm{H}$ NMR (400 MHz, acetone- $\left.d_{6}\right): \delta 8.42\left(\mathrm{H}^{1}, \mathrm{dd}, J=4.4,2.0 \mathrm{~Hz}, 1 \mathrm{H}\right), 8.27\left(\mathrm{H}^{3}, \mathrm{dd}\right.$, $J=8.0,1.6 \mathrm{~Hz}, 1 \mathrm{H}), 8.03\left(\mathrm{H}^{8}, \mathrm{dd}, J=8.0,1.2 \mathrm{~Hz}, 1 \mathrm{H}\right), 7.97\left(\mathrm{H}^{4}, \mathrm{~d}, J=8.8 \mathrm{~Hz}, 1 \mathrm{H}\right), 7.84\left(\mathrm{H}^{5}, \mathrm{~d}\right.$, $J=8.8 \mathrm{~Hz}, 1 \mathrm{H}), 7.73\left(\mathrm{H}^{7}, \mathrm{dd}, J=8.0,7.2 \mathrm{~Hz}, 1 \mathrm{H}\right), 7.51\left(\mathrm{H}^{6}, \mathrm{dd}, J=7.2,1.2 \mathrm{~Hz}, 1 \mathrm{H}\right), 7.43\left(\mathrm{H}^{2}\right.$, $\mathrm{dd}, J=8.0,4.4 \mathrm{~Hz}, 1 \mathrm{H}), 7.26\left(\mathrm{H}^{10}\right.$, app. t, $\left.J=8.0 \mathrm{~Hz}, 1 \mathrm{H}\right), 6.89\left(\mathrm{H}^{11}\right.$, ddd, $J=8.0,2.4,1.2 \mathrm{~Hz}$, $1 \mathrm{H}), 6.87-6.82\left(\mathrm{H}^{9}\right.$ and $\mathrm{H}^{13}$, multiple peaks, $\left.2 \mathrm{H}\right), 3.77\left(\mathrm{H}^{12}, \mathrm{~s}, 3 \mathrm{H}\right) ;{ }^{13} \mathrm{C} \mathrm{NMR}(100 \mathrm{MHz}$, acetone- $\left.d_{6}\right): \delta 160.12,148.86,147.76,147.48,142.54,136.30,136.04,132.09,129.78,129.16$, 129.05, 129.01, 128.28, 128.00, 127.07, 122.30, 122.20, 115.19, 112.00, 55.43. HRMS ESI with Formic Acid (m/z): $[\mathrm{M}+\mathrm{H}]^{+}$calcd for $\mathrm{C}_{20} \mathrm{H}_{16} \mathrm{NO}, 286.1232$; found, 286.1220.

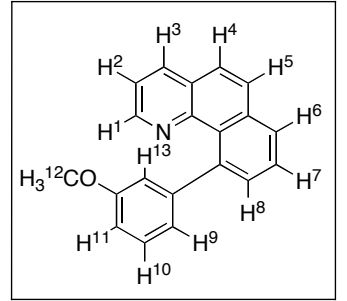

\section{Confirmation of Regioselectivity (assignments based on COSY and HSQC)}

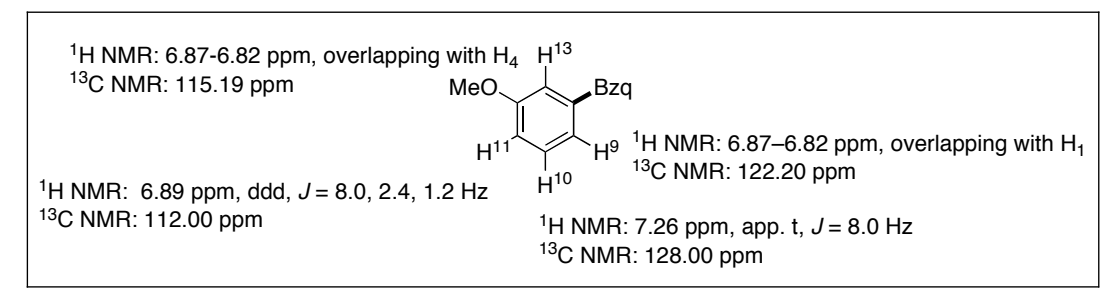

para isomer: ${ }^{1} \mathrm{H}$ NMR (400 MHz, acetone- $\left.d_{6}\right): \delta 8.43\left(\mathrm{H}^{1}, \mathrm{dd}, J=4.2,1.8 \mathrm{~Hz}, 1 \mathrm{H}\right), 8.26\left(\mathrm{H}^{3}\right.$, dd, $J=8.0,2.0 \mathrm{~Hz}, 1 \mathrm{H}), 7.99\left(\mathrm{H}^{8}, \mathrm{dd}, J=7.9,1.4 \mathrm{~Hz}, 1 \mathrm{H}\right), 7.95\left(\mathrm{H}^{4}, \mathrm{~d}, J=8.8 \mathrm{~Hz}, 1 \mathrm{H}\right), 8.82,\left(\mathrm{H}^{5}, \mathrm{~d}\right.$, $J=8.8 \mathrm{~Hz}, 1 \mathrm{H}), 7.71\left(\mathrm{H}^{7}, \mathrm{t}, J=7.6 \mathrm{~Hz}, 1 \mathrm{H}\right), 7.49\left(\mathrm{H}^{6}, \mathrm{dd}, J=7.2,1.6 \mathrm{~Hz}, 1 \mathrm{H}\right), 7.43\left(\mathrm{H}^{2}, \mathrm{dd}, J=\right.$ 8.0, 4.4 Hz, 1H), $7.20\left(\mathrm{H}^{9}, \mathrm{~d}, J=8.8 \mathrm{~Hz}, 2 \mathrm{H}\right), 6.92\left(\mathrm{H}^{10}, \mathrm{~d}, J=8.8 \mathrm{~Hz}, 2 \mathrm{H}\right), 3.86\left(\mathrm{H}^{11}, \mathrm{~s}, 3 \mathrm{H}\right)$; ${ }^{13} \mathrm{C}$ NMR (100 MHz, acetone- $\left.d_{6}\right): \delta$ 159.02, 147.74, 147.68, 142.54, 139.78, 136.34, 136.19, $132.56,130.63,129.92,129.25,128.80,128.27,128.03,127.01,122.27,113.51,55.49$. HRMS ESI with Formic Acid (m/z): [M+H] $]^{+}$calcd for $\mathrm{C}_{20} \mathrm{H}_{16} \mathrm{NO}, 286.1232$; found, 286.1224. 


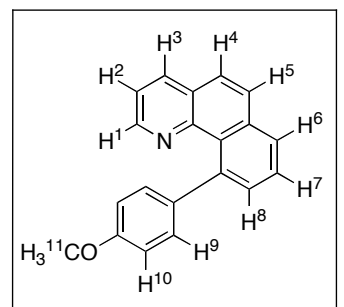

\section{Confirmation of Regioselectivity (assignments based on COSY and HSQC)}
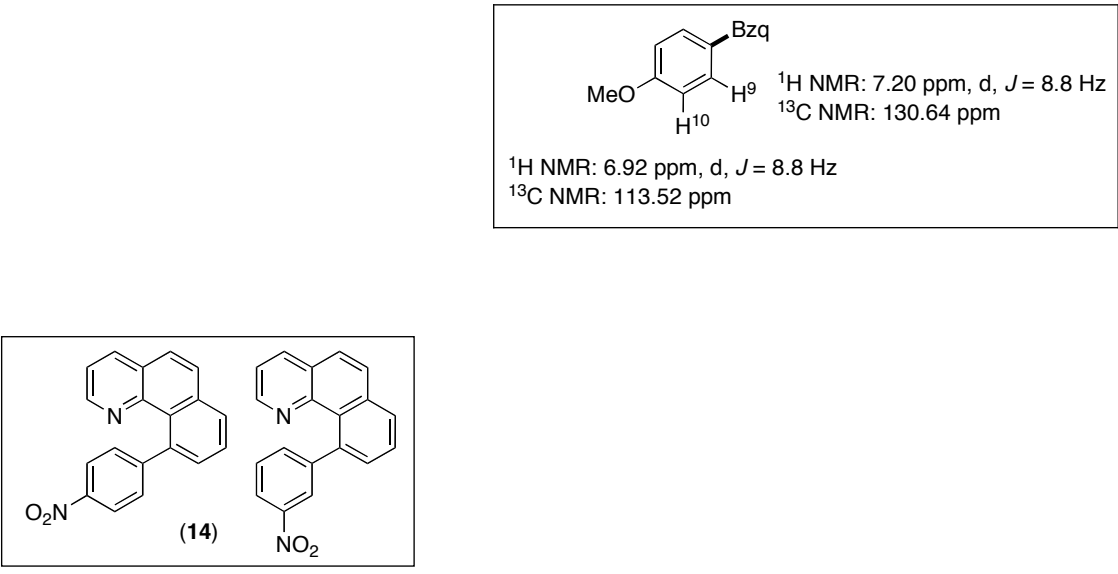

Product 14: $\mathrm{L} \sim \mathrm{C}_{\mathrm{Ar}}-\mathrm{H}=7,8$-benzo[ $\left.h\right]$ quinoline; $\mathrm{Ar}-\mathrm{H}=$ nitrobenzene; temperature $=130{ }^{\circ} \mathrm{C}$. The products were isolated as a mixture of isomers as a pale orange viscous oil ( $\mathrm{rf}=0.13 \mathrm{in} 95 \%$ hexanes $/ 5 \%$ EtOAc, $100 \mathrm{mg}, 77 \%$ yield, the $o: m: p$ ratio of $0: 3.5: 1$ was determined by GC analysis of crude reaction mixture). Trace amounts $(<5 \%)$ of the Bzq-Bzq dimer were observed in the ${ }^{1} \mathrm{H}$ NMR spectrum of the crude reaction mixture. The two regioisomers were separated by HPLC.

meta isomer: ${ }^{1} \mathrm{H} \mathrm{NMR}\left(400 \mathrm{MHz}, \mathrm{CDCl}_{3}\right): 8.36\left(\mathrm{H}^{1}, \mathrm{dd}, J=4.2,1.8 \mathrm{~Hz}, 1 \mathrm{H}\right), 8.25-8.21\left(\mathrm{H}^{11}\right.$ and $\mathrm{H}^{12}$, multiple peaks, $\left.2 \mathrm{H}\right), 8.12\left(\mathrm{H}^{3}, \mathrm{dd}, J=8.0,2.0 \mathrm{~Hz}, 1 \mathrm{H}\right), 8.00\left(\mathrm{H}^{8}, \mathrm{dd}, J=8.0,1.2 \mathrm{~Hz}, 1 \mathrm{H}\right)$. $7.89\left(\mathrm{H}^{4}, \mathrm{~d}, J=8.8 \mathrm{~Hz}, 1 \mathrm{H}\right), 7.74\left(\mathrm{H}^{5}, \mathrm{~d}, J=8.8 \mathrm{~Hz}, 1 \mathrm{H}\right), 7.72\left(\mathrm{H}^{7}, \mathrm{t}, J=7.6 \mathrm{~Hz}, 1 \mathrm{H}\right), 7.69\left(\mathrm{H}^{9}\right.$, $\mathrm{dt}, J=8.0,1.3 \mathrm{~Hz}, 1 \mathrm{H}), 7.54\left(\mathrm{H}^{10}, \mathrm{dd}, J=8.8,7.6 \mathrm{~Hz}, 1 \mathrm{H}\right), 7.52\left(\mathrm{H}^{6}, \mathrm{dd}, J=7.6,1.4 \mathrm{~Hz}, 1 \mathrm{H}\right)$, $7.35\left(\mathrm{H}^{2}, \mathrm{dd}, J=8.0,4.4 \mathrm{~Hz}, 1 \mathrm{H}\right) ;{ }^{13} \mathrm{C} \mathrm{NMR}\left(100 \mathrm{MHz}, \mathrm{CDCl}_{3}\right): \delta 147.89,147.70,146.88$, $146.14,138.91,135.52$, 135.07, 134.98, 131.18, 128.96, 128.65, 128.22, 127.98, 127.35, 127.13, 126.27, 123.93, 121.41, 120.80. HRMS ESI with Formic Acid (m/z): $[\mathrm{M}+\mathrm{H}]^{+}$calcd for $\mathrm{C}_{20} \mathrm{H}_{16} \mathrm{NO}, 286.1232$; found, 286.1220. 


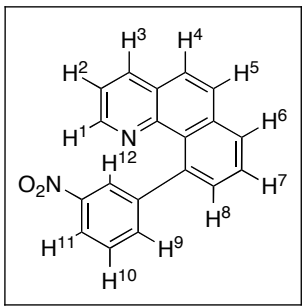

\section{Confirmation of Regioselectivity}

\section{(assignments based on COSY and chemical shift analysis)}

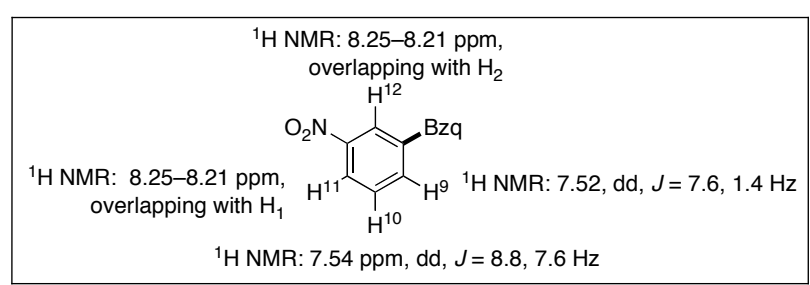

para isomer: ${ }^{1} \mathrm{H}$ NMR (400 MHz, acetone- $\left.d_{6}\right): \delta 8.42\left(\mathrm{H}^{1}, \mathrm{dd}, J=3.4,1.4 \mathrm{~Hz}, 1 \mathrm{H}\right), 8.36\left(\mathrm{H}^{3}, \mathrm{dd}\right.$, $J=6.4,1.6 \mathrm{~Hz}, 1 \mathrm{H}), 8.30\left(\mathrm{H}^{10}, \mathrm{~d}, J=7.2 \mathrm{~Hz}, 2 \mathrm{H}\right), 8.17\left(\mathrm{H}^{8}, \mathrm{dd}, J=6.4,1.2 \mathrm{~Hz}, 1 \mathrm{H}\right), 8.06\left(\mathrm{H}^{4}, \mathrm{~d}\right.$, $J=7.2 \mathrm{~Hz}, 1 \mathrm{H}), 7.94\left(\mathrm{H}^{5}, \mathrm{~d}, J=7.2 \mathrm{~Hz}, 1 \mathrm{H}\right), 7.84\left(\mathrm{H}^{7}, \mathrm{dd}, J=6.2,5.8 \mathrm{~Hz}, 1 \mathrm{H}\right), 7.57\left(\mathrm{H}^{9}\right.$ and $\mathrm{H}^{6}$, multiple peaks, $3 \mathrm{H}), 7.51\left(\mathrm{H}^{2}\right.$, dd, $\left.J=6.4,3.2 \mathrm{~Hz}, 1 \mathrm{H}\right) .{ }^{13} \mathrm{C} \mathrm{NMR}\left(100 \mathrm{MHz}, \mathrm{CDCl}_{3}\right): \delta 153.71$, $146.84,146.13,145.93,139.21,135.56,134.92,130.69,129.53,128.98,128.21,127.38,127.15$, 126.28, 122.82, 121.47 (a seventeenth signal was not resolved due to too little sample or overlapping signals).

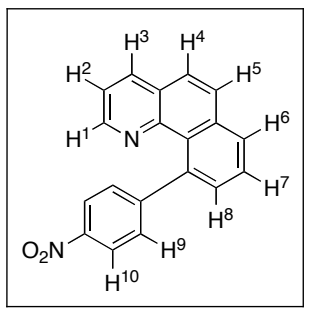

\section{Confirmation of Regioselectivity}
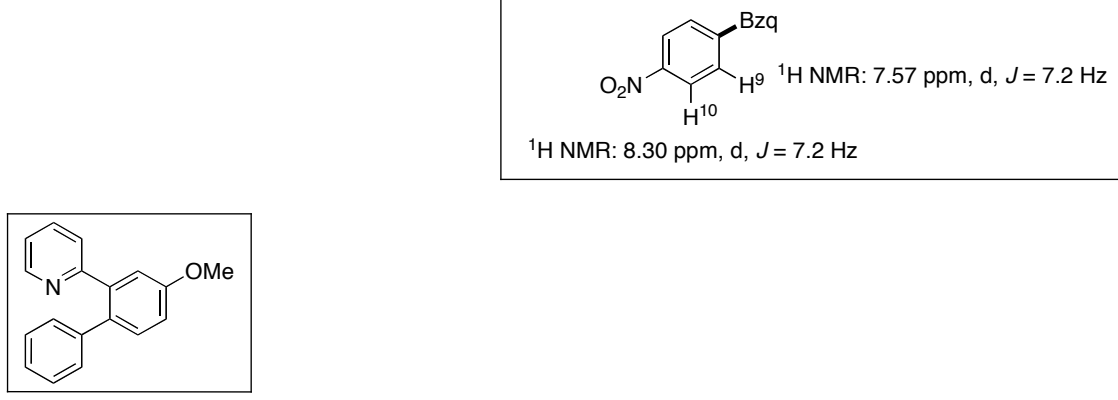
$\mathrm{L} \sim \mathrm{C}_{\mathrm{Ar}}-\mathrm{H}=2$ - $(m$-methoxyphenyl $)$ pyridine; $\mathrm{Ar}-\mathrm{H}=$ benzene; temperature $=150{ }^{\circ} \mathrm{C}$. The product was isolated as a pale orange viscous oil $(52 \mathrm{mg}, 46 \%$ yield, $\mathrm{rf}=0.05$ in $90 \%$ hexanes $/ 10 \%$ EtOAc). ${ }^{1} \mathrm{H}$ NMR $\left(400 \mathrm{MHz}\right.$, acetone- $\left.d_{6}\right): \delta 8.58\left(\mathrm{H}^{1}\right.$, ddd, $\left.J=3.6,1.2,0.8 \mathrm{~Hz}, 1 \mathrm{H}\right), 7.46\left(\mathrm{H}^{3}\right.$, td, $J=6.0,1.5 \mathrm{~Hz}, 1 \mathrm{H}), 7.35\left(\mathrm{H}^{7}, \mathrm{~d}, J=6.8 \mathrm{~Hz}, 1 \mathrm{H}\right), 7.25-7.17\left(\mathrm{H}^{8}, \mathrm{H}^{9}, \mathrm{H}^{10}\right.$, multiple peak, $\left.5 \mathrm{H}\right)$, 7.11-7.05 $\left(\mathrm{H}^{2}, \mathrm{H}^{4}, \mathrm{H}^{6}\right.$, multiple peaks, $\left.3 \mathrm{H}\right), 6.92\left(\mathrm{H}^{5}, \mathrm{dt}, J=5.2,0.8 \mathrm{~Hz}, 1 \mathrm{H}\right), 3.88\left(\mathrm{H}^{6}, \mathrm{~s}, 3 \mathrm{H}\right)$; ${ }^{13} \mathrm{C}$ NMR (100 MHz, $\left.\mathrm{CDCl}_{3}\right): \delta$ 160.04, 160.00, 150.30, 142.29, 141.86, 136.10, 134.10, 132.54, 130.52, 128.93, 127.22, 126.03, 122.51, 116.55, 115.14, 57.80. HRMS ESI with Formic Acid $(\mathrm{m} / \mathrm{z}):[\mathrm{M}+\mathrm{H}]^{+}$calcd for $\mathrm{C}_{18} \mathrm{H}_{16} \mathrm{NO} 262,1232$; found, 262.1227 .
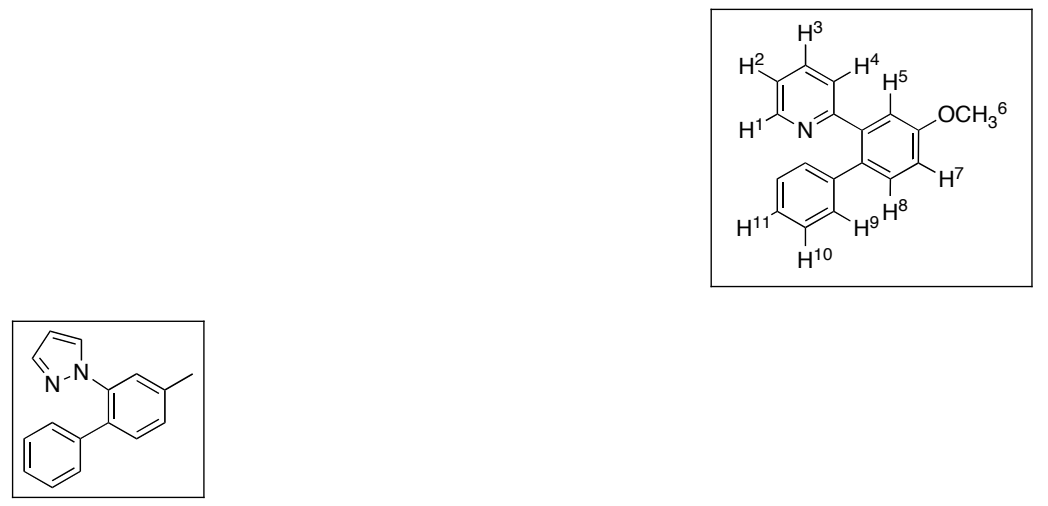

$\mathrm{L} \sim \mathrm{C}_{\mathrm{Ar}}-\mathrm{H}=1$-( $m$-tolyl)pyrazole $) ; \mathrm{Ar}-\mathrm{H}=$ benzene; temperature $=130{ }^{\circ} \mathrm{C}$. The product was isolated as yellow oil ( $\mathrm{rf}=0.11 \mathrm{in} 95 \%$ hexanes $/ 5 \%$ EtOAc, $26 \mathrm{mg}, 26 \%$ yield). ${ }^{1} \mathrm{H}$ NMR (500 $\left.\mathrm{MHz}, \mathrm{CD}_{2} \mathrm{Cl}_{2}\right): \delta 7.59\left(\mathrm{H}^{3}, \mathrm{~d}, J=2.0 \mathrm{~Hz}, 1 \mathrm{H}\right), 7.40\left(\mathrm{H}^{4}, \mathrm{bs}, 1 \mathrm{H}\right), 7.37\left(\mathrm{H}^{7}, \mathrm{~d}, J=8.0 \mathrm{~Hz}, 1 \mathrm{H}\right)$, $7.30\left(\mathrm{H}^{6}\right.$, bd, $\left.J=8.0 \mathrm{~Hz}, 1 \mathrm{H}\right), 7.29-7.25\left(\mathrm{H}^{9}, \mathrm{H}^{10}\right.$, multiple peaks, $\left.3 \mathrm{H}\right), 7.13\left(\mathrm{H}^{1}, \mathrm{~d}, J=2.5 \mathrm{~Hz}\right.$, $1 \mathrm{H}$ ), 7.10-7.07 ( $\left.\mathrm{H}^{8}, \mathrm{~m}, 2 \mathrm{H}\right), 6.20\left(\mathrm{H}^{2}, \mathrm{t}, J=2.3 \mathrm{~Hz}, 1 \mathrm{H}\right), 2.45\left(\mathrm{H}^{5}, \mathrm{~s}, 3 \mathrm{H}\right) ; 20.94$ (two carbon peaks coincidentally overlap). HRMS ESI with Formic Acid (m/z): $[\mathrm{M}+\mathrm{H}]^{+}$calcd for $\mathrm{C}_{16} \mathrm{H}_{14} \mathrm{~N}_{2}$ 235.1235; found, 235.1232 .

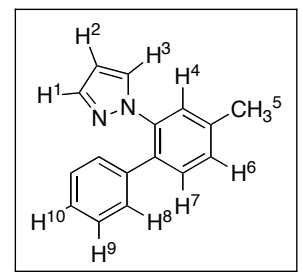




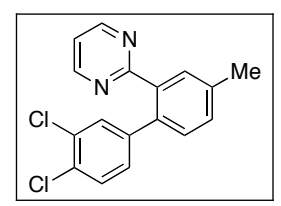

$\mathrm{L} \sim \mathrm{C}_{\mathrm{Ar}}-\mathrm{H}=2-m$-tolylpyrimidine; $\mathrm{Ar}-\mathrm{H}=$ benzene; temperature $=150{ }^{\circ} \mathrm{C}$. The product was isolated as white solid (93 $\mathrm{mg}, 69 \%$ yield, $\mathrm{mp}=132.7-135.8^{\circ} \mathrm{C}, \mathrm{rf}=0.09$ in $90 \%$ hexanes $/ 10 \%$ EtOAc). ${ }^{1} \mathrm{H}$ NMR $\left(500 \mathrm{MHz}\right.$, acetone- $\left.d_{6}\right): 8.69\left(\mathrm{H}^{2}, \mathrm{~d}, J=4.0 \mathrm{~Hz}, 2 \mathrm{H}\right), 7.70\left(\mathrm{H}^{3}, \mathrm{bs}, 1 \mathrm{H}\right), 7.41-$ $7.35\left(\mathrm{H}^{5}, \mathrm{H}^{6}, \mathrm{H}^{9}\right.$, multiple peaks, $\left.3 \mathrm{H}\right), 7.30\left(\mathrm{H}^{1}, \mathrm{t}, J=4.0 \mathrm{~Hz}, 2 \mathrm{H}\right), 7.28\left(\mathrm{H}^{9}, \mathrm{~d}, J=1.2 \mathrm{~Hz}, 1 \mathrm{H}\right)$, $6.98\left(\mathrm{H}^{7}, \mathrm{dd}, J=8.3,2.3 \mathrm{~Hz}, 1 \mathrm{H}\right), 2.46\left(\mathrm{H}^{4}, \mathrm{~s}, 3 \mathrm{H}\right) ;{ }^{13} \mathrm{C}$ NMR (125 MHz, acetone- $\left.d_{6}\right): 167.93$, $157.88,143.77,139.29,138.82$, 137.23, 132.49, 132.07, 131.77, 131.33, 131.01, 130.73, 130.63, 130.13, 120.03, 21.14. HRMS ESI with Formic Acid $(\mathrm{m} / \mathrm{z})$ : $[\mathrm{M}+\mathrm{H}]^{+}$calcd for $\mathrm{C}_{11} \mathrm{H}_{11} \mathrm{~N}_{2}$, 315.0456; found, 315.0447.
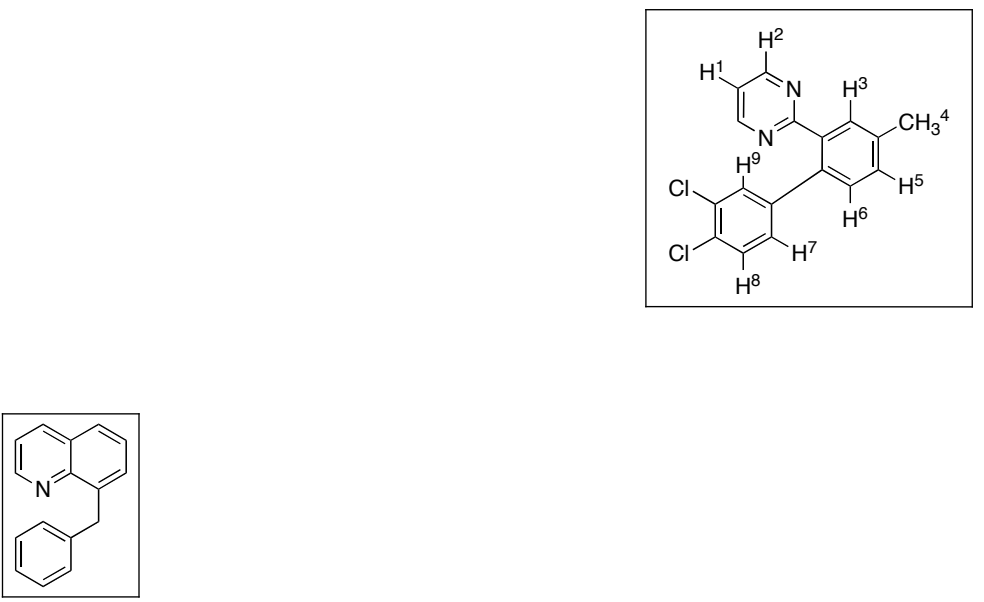

$\mathrm{L} \sim \mathrm{C}_{\mathrm{Ar}}-\mathrm{H}=8$-methylquinoline; $\mathrm{Ar}-\mathrm{H}=$ benzene; temperature $=150{ }^{\circ} \mathrm{C}$. The product was isolated as white solid ( $\mathrm{rf}=0.08 \mathrm{in} 98 \%$ hexanes $/ 2 \%$ EtOAc, $45 \mathrm{mg}, 48 \%$ yield). Spectroscopic data were identical to the literature data. ${ }^{6}$ 


\section{Mechanistic Studies}

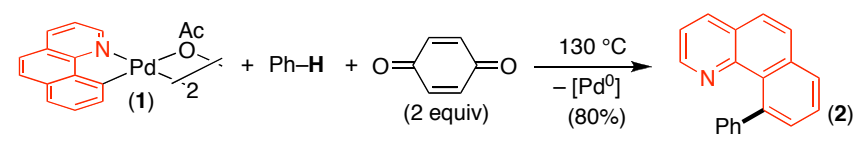

Palladacycle 1 [148 mg, $0.215 \mathrm{mmol}, 0.5$ equiv ( 1 equiv based on Pd)] and benzoquinone (47 $\mathrm{mg}, 0.43 \mathrm{mmol}, 1$ equiv) were vigorously stirred in $3.75 \mathrm{~mL}$ benzene at $130{ }^{\circ} \mathrm{C}$ for $12 \mathrm{~h}$. The reaction mixture was filtered through silica gel and washed with $150 \mathrm{~mL}$ ethyl acetate. Product 2 was isolated by column chromatography as a crystalline white solid ( $80 \mathrm{mg}, 73 \%$ yield).

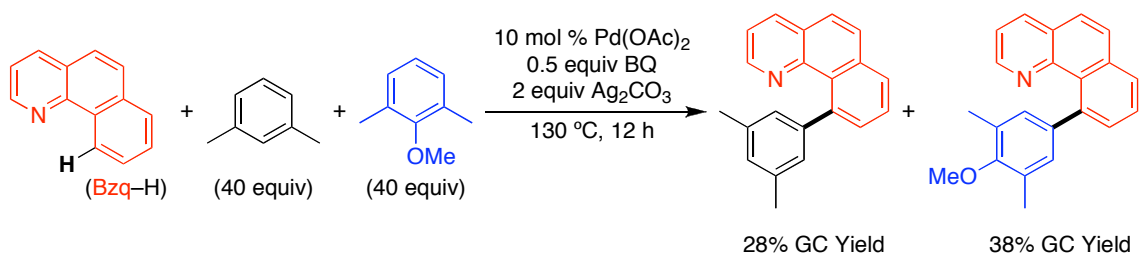

Used the general procedure for the reaction set up; $\mathrm{L} \sim \mathrm{C}_{\mathrm{Ar}}-\mathrm{H}=7,8$-benzo $[h]$ quinoline; Ar- $\mathrm{H}=$ 2,6-dimethylanisole (2.44 mL, $17.2 \mathrm{mmol}, 40$ equiv) and $m$-xylene (2.10 mL, $17.2 \mathrm{mmol}, 40$ equiv); temperature $=150{ }^{\circ} \mathrm{C}$. Upon cooling, methyl naphthalene was added as an internal standard, and the corrected yields were determined by GC analysis.

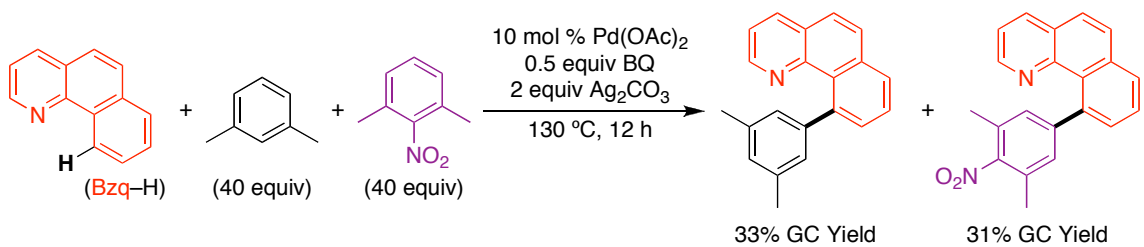

Used the general procedure for the reaction set up; $\mathrm{L} \sim \mathrm{C}_{\mathrm{Ar}}-\mathrm{H}=7,8$-benzo $[h]$ quinoline; $\mathrm{Ar}-\mathrm{H}=2$ nitro- $m$-xylene (2.34 mL, $17.2 \mathrm{mmol}, 40$ equiv) and $m$-xylene (2.10 mL, $17.2 \mathrm{mmol}, 40$ equiv); temperature $=150{ }^{\circ} \mathrm{C}$. Upon cooling, methyl naphthalene was added as an internal standard, and the corrected yields were determined by GC analysis. 

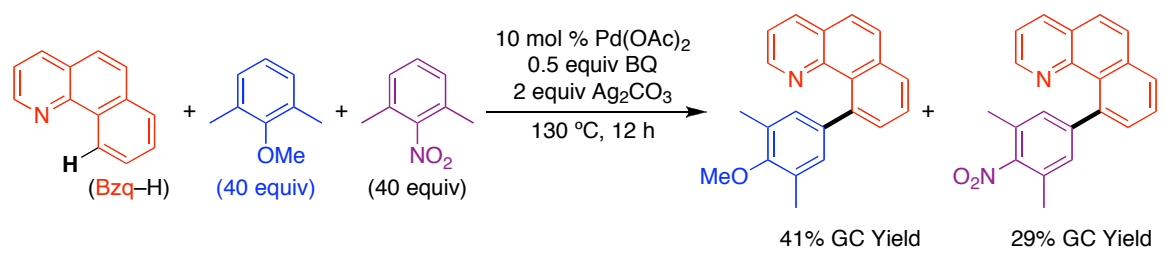

Used the general procedure for the reaction set up; $\mathrm{L} \sim \mathrm{C}_{\mathrm{Ar}}-\mathrm{H}=7,8$-benzo[ $[h]$ quinoline; $\mathrm{Ar}-\mathrm{H}=2$ nitro- $m$-xylene (2.34 mL, $17.2 \mathrm{mmol}, 40$ equiv) and 2,6-dimethylanisole (2.44 mL, $17.2 \mathrm{mmol}$, 40 equiv); temperature $=150{ }^{\circ} \mathrm{C}$. Upon cooling, methyl naphthalene was added as an internal standard, and the corrected yields were determined by GC analysis.

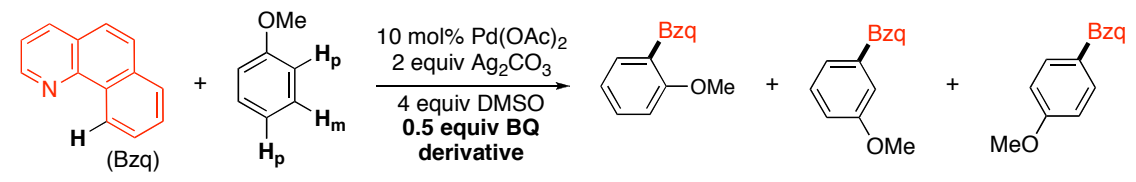

\begin{tabular}{|c|c|c|c|}
\hline Entry & Olefin Derivative & GC Yield & $o: m: p$ \\
\hline 1 & benzoquinone & $88 \%$ & $1: 2.9: 3.3$ \\
2 & 2-methylbenzoquinone & $81 \%$ & $1: 5.9: 4.4$ \\
3 & 2,6-dimethylbenzoquinone & $51 \%$ & $1: 15.9: 6.7$ \\
4 & duroquinone & $35 \%$ & $1: 18.1: 6.5$ \\
\hline
\end{tabular}

Used the general procedure for the reaction setup on $1 / 5$ the scale; $\mathrm{L} \sim \mathrm{C}_{\mathrm{Ar}}-\mathrm{H}=7,8$ benzo[ $h]$ quinoline; $\mathrm{Ar}-\mathrm{H}=$ anisole; temperature $=130{ }^{\circ} \mathrm{C}$. Upon cooling, methyl naphthalene was added as an internal standard, and the corrected yields were determined by GC analysis. 


\section{REFERENCES:}

1. Harris, R. K.; Becker, E. D.; Cabral De Menezes, S. M.; Goodfellow, R.; Granger, P. Pure Appl. Chem. 2001, 73, 1795.

2. Cristau, H-J.; Cellier, P. P.; Spindler, J-F.; Taillefer, M. Eur. J. Org. Chem. 2004, 695.

3. Littke, A. F.; Dai, G. C. J. Am. Chem. Soc. 2000, 122, 4020.

4. Dick, A. R.; Hull, K. L.; Sanford, M. S. J. Am. Chem. Soc. 2004, 126, 2300.

5. Dick, A. R. Ph.D. Thesis, University of Michigan, Ann Arbor, MI, 2007.

6. Kalyani, D.; Deprez, N. R.; Desai, L. V.; Sanford, M. S. J. Am. Chem. Soc. 2005, 127, 7330. 